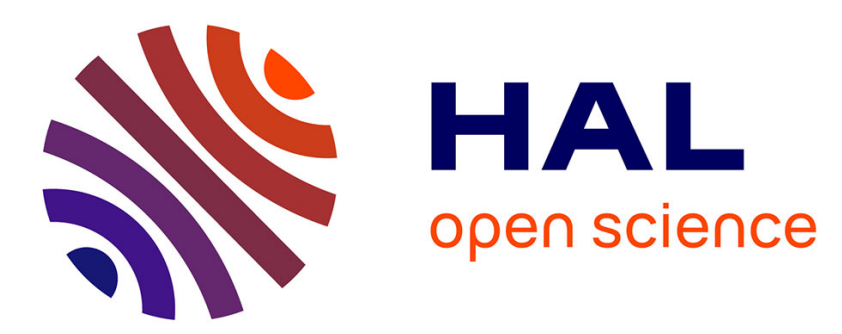

\title{
New properties of the V-line Radon transform and their imaging applications
}

\author{
T. T. Truong, M. K. K Nguyen
}

\section{To cite this version:}

T. T. Truong, M. K. K Nguyen. New properties of the V-line Radon transform and their imaging applications. Journal of Physics A: Mathematical and Theoretical, 2015, 48(2015) 405204, 10.1088/17518113/48/40/405204. hal-01260337

\section{HAL Id: hal-01260337 \\ https://hal.science/hal-01260337}

Submitted on 13 Apr 2018

HAL is a multi-disciplinary open access archive for the deposit and dissemination of scientific research documents, whether they are published or not. The documents may come from teaching and research institutions in France or abroad, or from public or private research centers.
L'archive ouverte pluridisciplinaire HAL, est destinée au dépôt et à la diffusion de documents scientifiques de niveau recherche, publiés ou non, émanant des établissements d'enseignement et de recherche français ou étrangers, des laboratoires publics ou privés. 


\title{
New properties of the V-line Radon transform and their imaging applications
}

\author{
T. T. Truong $\dagger \dagger$ and M.K. Nguyen* \\ † Laboratoire de Physique Théorique et Modélisation \\ (CNRS UMR 8089/Université de Cergy-Pontoise), \\ F-95302 Cergy-Pontoise Cedex, France. \\ *Equipes de Traitement de l'Information et Systèmes (ETIS), \\ ENSEA/ Université de Cergy-Pontoise/ UMR CNRS 8051 \\ F-95014 Cergy-Pontoise Cedex, France
}

\begin{abstract}
This paper reports on new aspects of the so-called V-line Radon transforms complementing those reported in an earlier work. These new properties are nicely uncovered and described with Cartesian coordinates. In particular, we show that the V-line Radon transform belongs to the class of Radon transforms on curves in the plane which can be mapped onto the standard Radon transform on straight lines and thereby are fully characterizable and invertible. Next, we show that the effect of geometric inversion on the V-line Radon transform is to produce a new Radon transform on a pair of supplementary circular arcs, which provides a new access to image reconstruction in the so-called Norton's modality of Compton scatter tomography, a front runner in the race for alternatives to current emission imaging.
\end{abstract}

\section{Introduction}

The integral transform introduced by J Radon in 1917 [1], has turned out to be a corner stone for integral geometry in the sense of I M Gelfand. Over the years, it has provided prolific applications in many disciplines and fields of mathematics, physics and imaging science. Its main property lies in the possibility to reconstruct a function on $\mathbb{R}^{2}$ from the knowledge of its integral "profiles" (or "projections") along straight lines drawn in a plane. It has contributed to the advancement in mathematics (e.g. Partial Differential Equations [2], probabilities [3], group theory [4], differential geometry [5]). In physics, it has opened a new investigation path to quantum states in phase space [6,7], to particle dynamics in accelerators [8]. In imaging technologies, it has covered medical imaging [9], astrophysics [10], non-destructive control [11], geophysical prospection [12], etc. As far as modalities are concerned, it has served in transmission imaging (Computerized Tomography) as well as in emission tomography (in Single Photon Computerized Tomography (SPECT) and in Positron Emission Tomography (PET)).

$\dagger$ To whom correspondence should be addressed (truong@u-cergy.fr) 
Over the years, the idea of extending the Radon transform on straight lines to Radon transforms on generalized linear paths has come up under the form of the integration of a function in $\mathbb{R}^{2}$ along two branches of straight lines meeting at a common point. This new geometric figure was called first a V-line but later on the name of Broken-line has occurred also in the literature.

The concept of a V-line path has been introduced first by R. Basko, with the intent to model the imaging process of a two-dimensional Compton camera [13]. This is a figure having the shape of a capital $\mathrm{V}$ letter, with a swinging symmetry axis, with its vertex $M$ moving on a fixed straight line and with variable opening angle $\omega$. This type of $\mathrm{V}$-line depends on two parameters: the position of $M$ on the fixed line and the angle $\omega$. The problem of inverting the Radon transform of a function on the set of such V-lines has been a challenge for a long time but seems to have been solved very recently [14]. A particular class of $\mathrm{V}$-lines with fixed symmetry axis direction has been introduced a bit later in two-dimensional SPECT imaging [15, 16]. The basic properties of this Radon transform have been discussed in [17]. Recently a new class of Radon transform on cones of fixed axis direction and constant opening angle has been considered and inverted [18] in two and three dimensions.

In the last few years, investigations on Single Scattering Optical Tomography (SSOT) have brought in the concept of Broken-line Radon transform by [19]. This figure has also the shape of a V-line. But its opening angle is fixed and it moves rigidly in $\mathbb{R}^{2}$. Of course the displacement in the plane depends on two parameters, which may be chosen as the two coordinates of its vertex. The inversion of this transform is new and has been solved in $[20,21]$. It has stirred up more interest and discussions in $[22,23]$ and has led to wider generalizations with other detection geometries and inclusion of attenuation effects by $[24,25]$ as well as with multi-lines intersecting a vertex, under the name of Star Transform in [26].

The aim here is to present some new properties and aspects of the Radon transform on a class of $\mathrm{V}$-lines with symmetry axis direction parallel to the $O y$ axis and vertex on the $O x$-axis, to complement those given in a preceding paper by the authors [17]. To make the paper accessible to a wider audience, we shall use a formulation in cartesian coordinates. In section 2, we first review some properties of the standard Radon transform and describe some aspects which appear in the use of Cartesian coordinates. Then we discuss a two-dimensional version of the socalled X-ray transform in section 3, in which a function is integrated on half-lines in $\mathbb{R}^{2}$ starting from the $O x$-axis. The next section 4 , deals with new properties of the V-line Radon transform. The main result is that it can be converted to the standard Radon transform on the set of functions that symmetric with respect to the reflection $y \rightarrow-y$. We show that the inversion procedure is derivable from this identification. Section 5 discusses geometric inversion in the plane and its effects on the generalized linear path Radon transforms. It gives explicit connecting formulas to Radon transforms on full circles, upper circular arcs and pairs of upper circular arcs. In section 6 , we show that the S J Norton modality for Compton scattering tomography, introduced in 1995, can be adequately handled by these new Radon transforms on circular arcs, in particular with a new scanning protocol. Conclusion and future work perspectives are given in the last section 7 . 


\section{The classical line Radon transform revisited}

\subsection{Definition}

The aim of this section is to recall the properties of the classical line Radon transform (RT) in cartesian coordinates, which shall be helpful to understand the V-line Radon transform (VRT). This type of formulation has been known before in the seismic community under the name of slant stacks[12].

Traditionally, the RT maps smooth integrable function $f(x, y)$ in $\mathbb{R}^{2}$ (hereafter called object function) to its integral $\mathcal{R} f(s, \phi)$ on lines given by their canonical equation $s=x \cos \phi+y \sin \phi$ (hereafter $\mathcal{R} f(s, \phi)$ is called image function), where $s$ is the distance of the coordinate origin to the line and $\phi$ is the angle made by the line unit normal vector with the $O x$-axis and assumed to be $\phi \neq \pi / 2$, see Fig. 4

$$
f(x, y) \rightarrow \mathcal{R} f(s, \phi)=\int_{\mathbb{R}^{2}} d x d y \delta(s-x \cos \phi-y \sin \phi) f(x, y),
$$

where $(s, \phi) \in=(\mathbb{R},[0, \pi])$ (hereafter called standard RT space). For the properties of $\mathcal{R} f(s, \phi)$ in terms of functions spaces to which $f(x, y)$ belongs, we refer the reader to the appropriate literature, e.g. [9].

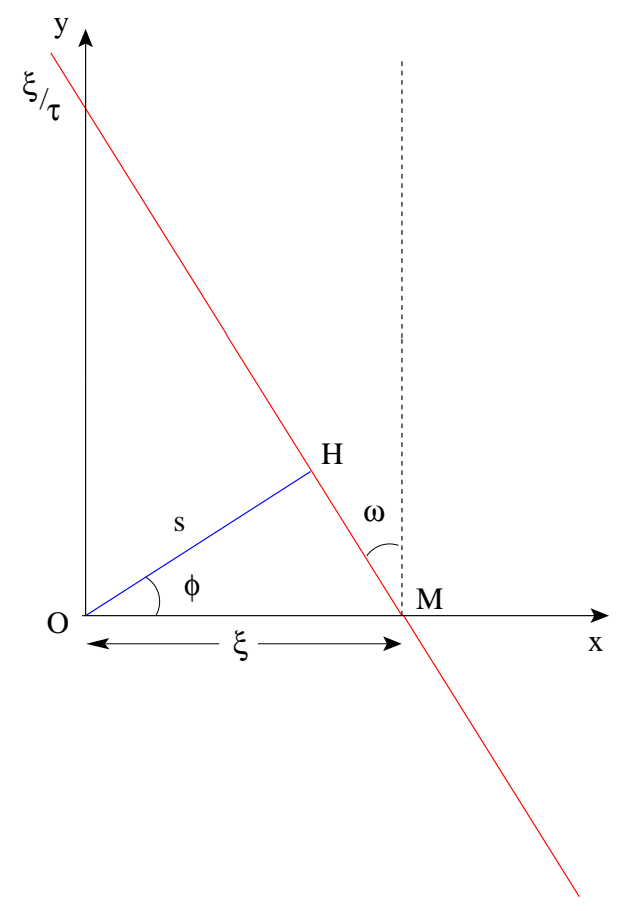

Figure 1. Radon transform 


\subsection{Cartesian coordinates}

From the defining equation (1), an expression in terms of a function given in polar coordinates as $f(r, \theta)$ may be easily derived

$$
\mathcal{R} f(s, \phi)=\int_{s}^{\infty} \frac{d r}{\sqrt{1-\frac{s^{2}}{r^{2}}}}\left[f\left(r, \phi+\cos ^{-1} \frac{s}{r}\right)+f\left(r, \phi-\cos ^{-1} \frac{s}{r}\right)\right] .
$$

Now in a Cartesian coordinate system $(x, y) \in \mathbb{R}^{2}$ (called object space), the straight line of equation $s=x \cos \phi+y \sin \phi$, for $\phi \neq \pi / 2$, takes the form $x-\xi+y \tau=0$, via the following change of variables

$$
\tau=\tan \phi, \quad \xi=\frac{s}{\cos \phi} \quad \text { and } \quad d \xi d \tau=\frac{1}{\cos ^{3} \phi} d s d \phi .
$$

Conversely, one has also

$$
\phi=\tan ^{-1} \tau, \quad s=\frac{\xi}{\sqrt{1+\tau^{2}}} \quad \text { and } \quad d s d \phi=\frac{1}{\left(1+\tau^{2}\right)^{3 / 2}} d \xi d \tau .
$$

Since $(s, \phi) \in(\mathbb{R},[0, \pi])$, this means that $(\xi, \tau) \in \mathbb{R}^{2}$ (hereafter called Cartesian RT space), as well as $(x, y) \in \mathbb{R}^{2}$.

Equation (1) is replaced by

$$
\mathcal{R} f(\xi, \tau)=\sqrt{1+\tau^{2}} \int_{\mathbb{R}^{2}} d x d y \delta(x-\xi+y \tau) f(x, y)
$$

Its adjoint $\mathbb{R}^{\dagger}$ acts on functions $g(\xi, \tau)$ as integrals $\mathcal{R}^{\dagger} g(x, y)$ concentrated on straight lines of equation $\xi-x-y \tau=0$, given by

$$
\begin{aligned}
\mathcal{R}^{\dagger} g(x, y) & =\int_{\mathbb{R}^{2}} \frac{d \xi d \tau}{\left(1+\tau^{2}\right)^{3 / 2}} \sqrt{1+\tau^{2}} \delta(x-\xi+y \tau) g(\xi, \tau) \\
& =\int_{-\infty}^{\infty} \frac{d \tau}{1+\tau^{2}} g(x+y \tau, \tau) .
\end{aligned}
$$

Equation (6) may be viewed as the RT in $(\xi, \tau)$ space of function of the form $g(\xi, \tau) /\left(1+\tau^{2}\right)^{3 / 2}$.

The known inversion formula ( see e.g.[9]) reads now

$$
f(x, y)=-\frac{1}{2 \pi^{2}} \int_{-\infty}^{\infty} \frac{d \tau}{\sqrt{1+\tau^{2}}} \text { P.V. } \int_{-\infty}^{\infty} d \xi \frac{1}{(\xi-x-y \tau)} \frac{\partial}{\partial \xi} \mathcal{R} f(\xi, \tau),
$$

where P.V. designates the principal value of the integral to be carried out.

Proposition 2.1 The product $\mathcal{R}^{\dagger} \mathcal{R}$ is represented by the kernel

$$
\mathcal{R}^{\dagger} \mathcal{R}\left(x^{\prime}, y^{\prime} \mid x, y\right)=\frac{1}{\sqrt{\left(x-x^{\prime}\right)^{2}+\left(y-y^{\prime}\right)^{2}}}, \quad(x, y) \in \mathbb{R}^{2}, \text { and }\left(x^{\prime}, y^{\prime}\right) \in \mathbb{R}^{2} \text {. }
$$

Proof. By definition of the product of two integral operators, we have

$$
\mathcal{R}^{\dagger} \mathcal{R}\left(x^{\prime}, y^{\prime} \mid x, y\right)=\int_{\mathbb{R}^{2}} \frac{d \xi d \tau}{\left(1+\tau^{2}\right)^{3 / 2}} \sqrt{1+\tau^{2}} \delta(x-\xi+y \tau) \sqrt{1+\tau^{2}} \delta\left(x^{\prime}-\xi+y^{\prime} \tau\right) .
$$

$\xi$-integration yields

$$
\mathcal{R}^{\dagger} \mathcal{R}\left(x^{\prime}, y^{\prime} \mid x, y\right)=\int_{-\infty}^{\infty} \frac{d \tau}{\sqrt{1+\tau^{2}}} \delta\left(\left(x^{\prime}-x\right)+\tau\left(y^{\prime}-y\right)\right) .
$$


Finally the last $\tau$-integration gives

$$
\mathcal{R}^{\dagger} \mathcal{R}\left(x^{\prime}, y^{\prime} \mid x, y\right)=\frac{1}{\sqrt{\left(x-x^{\prime}\right)^{2}+\left(y-y^{\prime}\right)^{2}}} .
$$

- This formula has been established in the standard RT in $(s, \phi)$-coordinates by [9].

Remark 2.2 H H Barrett in [9] has pointed out that this property of $\mathcal{R}^{\dagger} \mathcal{R}$ gives rise to an alternative inversion formula for RT, by noting that the two-dimensional Fourier transform of the object function $\widetilde{f}(p, q)$ may be obtained as

$$
\tilde{f}(p, q)=\sqrt{p^{2}+q^{2}} \int_{\mathbb{R}^{2}} d x d y e^{-2 i \pi(p x+q y)} \mathcal{R}^{\dagger}(\mathcal{R} f)(x, y) .
$$

$f(x, y)$ is recovered as

$$
f(x, y)=\int_{\mathbb{R}^{2}} d p d q e^{2 i \pi(p a x+q y)} \widetilde{f}(p, q) .
$$

\subsection{Some features of RT images in Cartesian coordinates}

2.3.1. Image of a point source A unit point source localized at $\left(x_{0}, y_{0}\right)$, is represented by the object function $f(x, y)=\delta\left(x-x_{0}\right) \delta\left(y-y_{0}\right)$. Its Cartesian RT image is the kernel $\sqrt{1+\tau^{2}} \delta\left(\xi-x_{0}-y_{0} \tau\right)$. Thus in Cartesian RT space $(\xi, \tau)$, the support of this image is simply a straight line of equation $\xi-x_{0}-y_{0} \tau=0$ in a Cartesian coordinate system $(\tau, \xi)$. Recall that in RT image coordinate system $(s, \phi)$, it is a sinogram. And such families of lines, which are structurally simpler, are easier to handle than families of sinograms in some applications.

2.3.2. Image of an object supported by a curve This object may be considered as a one-dimensional succession of point sources, with varying strength, lying on a smooth curve. Hence its Cartesian RT image support is a one-parameter family of straight lines. Under some conditions, these lines admit an envelop curve in the $(\xi, \tau)$-plane $[27]$.

As an illustration, we may consider an object of unit strength supported by a circle of radius $R$ and centered at O. The object function is thus $f(x, y)=\delta\left(R-\sqrt{x^{2}+y^{2}}\right)$. By definition its Cartesian RT transform is

$$
\mathcal{R} f(\xi, \tau)=\sqrt{1+\tau^{2}} \int_{\mathbb{R}^{2}} d x d y \delta(\xi-x-y \tau) \delta\left(R-\sqrt{x^{2}+y^{2}}\right),
$$

which, after successive integrations, is

$$
\mathcal{R} f(\xi, \tau)=\frac{2}{\sqrt{1+\tau^{2}-\frac{\xi^{2}}{R^{2}}}} .
$$

This shows that $\mathcal{R} f(\xi, \tau)$ is supported in the set $\left(\frac{\xi^{2}}{R^{2}}\right)-\tau^{2}<1$, a domain limited by the two branches of a hyperbola but containing the coordinate origin O. Of course this function becomes singular on this hyperbola.

To see how the hyperbolic envelop arises, observe that the Cartesian RT image of a point source is a line of equation $\xi-x-y \tau=0$ and that the totality of points on the support curve generates a one-parameter set of lines $\xi-R \cos t-\tau R \sin t=0$ (the circle being represented by its parametric equations $x=R \cos t$ and $y=R \sin t$ ). 
Using elementary analysis, we see that this family of lines admits an envelop which is obtained by eliminating $t$ from the line equation and its $t$-derivative, i.e.

$$
\frac{d}{d t}(\xi-R \cos t-\tau R \sin t=0) \quad \text { or } \quad \tan t=\tau .
$$

To perform this elimination, we must express $\sin t$ and $\cos t$ in terms of $\tau$ according to the previous equation, which implies that $\tau \sin t$ and $\cos t$ should have always the same sign as a function of $\tau$. From this observation, one can see that the envelop is precisely the hyperbola $\left(\frac{\xi^{2}}{R^{2}}-\tau^{2}\right)=1$ delimiting the support of the Cartesian RT image containing the coordinate origin O. In the image support area, the straight lines support of images of separate point sources are tangent to the hyperbola, see Fig. 2

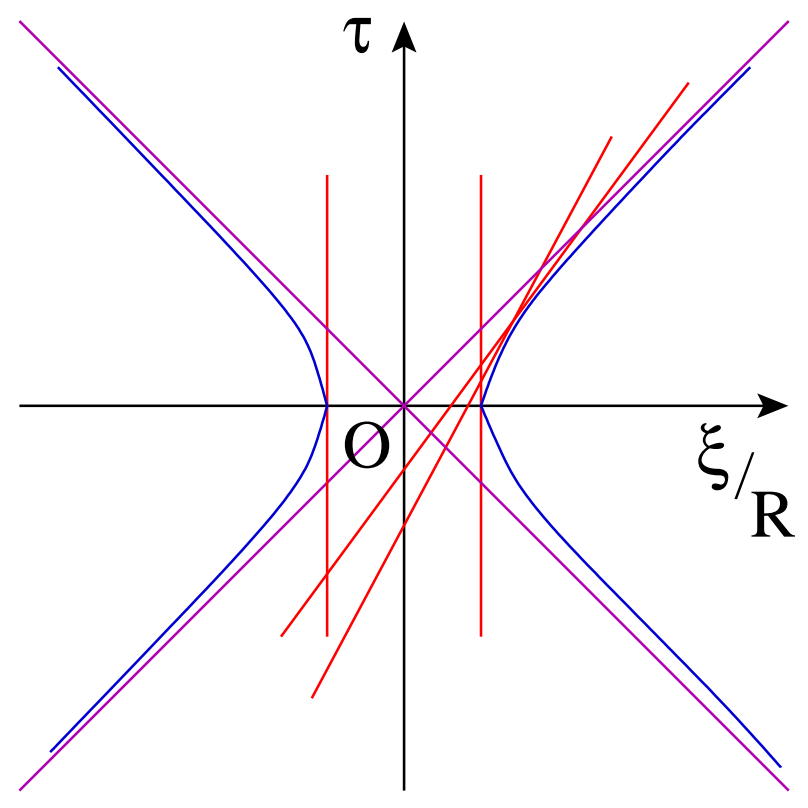

Figure 2. The hyperbola envelop

Remark 2.3 If the curve is a straight line going through the origin $y=\beta x$, then the envelop is degenerate and is reduced to a point or coordinates $(\xi, \tau)=(0,1 / \beta)$.

2.3.3. Image of a two-dimensional object A full two-dimensional object may be considered as a set of point sources of various strengths stacked inside a bounded contour of the object plane. Thus its Cartesian RT is computable and the image support in $(\xi, \tau)$ RT space is the union of all such lines. As an illustration, we may compute the Cartesian RT transform of the following simple function

$$
\begin{aligned}
f(x, y) & =1, \quad \text { for } \quad \sqrt{x^{2}+y^{2}}<1 \\
& =0 \quad \text { otherwise. }
\end{aligned}
$$

Its Cartesian RT transform can be readily seen as

$$
\begin{aligned}
\mathcal{R} f(\xi, \tau) & =2 \sqrt{\frac{R^{2}\left(1+\tau^{2}\right)-\xi^{2}}{1+\tau^{2}}} \quad \text { for } \quad 0<\xi<R \sqrt{1+\tau^{2}} \\
& =0 \quad \text { otherwise. }
\end{aligned}
$$


Clearly the support in $(\xi, \tau)$ RT space is the area delimited by the hyperbola $(\xi / R)^{2}-\tau^{2}=1$, containing the coordinate origin $\mathrm{O}$. In this area stands all the straight lines support of all point sources of the object, which may be thought as formed by concentric circular supports of radius less than $R$.

Remark 2.4 For a two-dimensional object function with a support that exhibits a texture formed by a one-parameter family of curves, its Cartesian RT may display a strong characteristic pattern in Cartesian $(\xi, \tau)-R T$ image space, susceptible to be observed and more easily detected. This property has been used in seismic exploration.

\subsection{Concept of back-projection of function reconstruction}

In this subsection, we examine the particular structure of the inverse RT formula to deduce some consequences. Equation (6) may be rewritten as

$$
f(x, y)=\int_{-\infty}^{\infty} \frac{d \tau}{1+\tau^{2}} g(x+y \tau, \tau)
$$

where

$$
g(x+y \tau, \tau)=-\frac{\sqrt{1+\tau^{2}}}{2 \pi^{2}} \text { P.V. } \int_{-\infty}^{\infty} d \xi \frac{1}{\xi-(x+y \tau)} \frac{\partial}{\partial \xi} \mathcal{R} f(\xi, \tau) .
$$

We notice that $g(x+y \tau, \tau)$ as a function of $(x, y)$ takes the same value as long as $(x+y \tau)$ keeps having the same value. This means that it is constant on straight lines of equation $(x+y \tau)=\xi_{0}$, where $\xi_{0} \in \mathbb{R}$. This is an example of "plane wave" in the sense of F. John, see [2]. Another point of view consists in saying that the value $g\left(\xi_{0}, \tau\right)$ of the function $g(\cdot, \tau)$ has been back-projected on the straight line of equation $(x+y \tau)=\xi_{0}$ as $g(x+y \tau, \tau)$, by smearing or spreading the value $g\left(\xi_{0}, \tau\right)$ all along this straight line.

Equation (19) suggests then that to reconstruct $f(x, y)$ one may sum the back projected function $g(x+y \tau, \tau)$ over the integration measure $d \tau /\left(1+\tau^{2}\right)$. Of course, if $g(x+y \tau, \tau)$ is properly chosen or derived from an exact formula, one does correctly reconstruct $f(x, y)$. But if $g(x+y \tau, \tau)$ arises as measured data or is given by some procedure, then this $\tau$-integration yields a "crude" reconstruction of $f(x, y)$. To illustrate this point, we may consider the back-projection of the exact data of a point source or Point Spread Function (PSF) at site $\left(x_{0}, y_{0}\right)$, i.e.

$$
\sqrt{1+\tau^{2}} \delta\left(\xi-x_{0}-y_{0} \tau\right) \rightarrow \sqrt{1+\tau^{2}} \delta\left(x+y \tau-x_{0}-y_{0} \tau\right)
$$

This back-projected function is now integrated over $d \tau$ to reconstruct crudely the $f(x, y)$ of a point source as

$$
f(x, y) \approx \int_{-\infty}^{\infty} \frac{d \tau}{1+\tau^{2}} \sqrt{1+\tau^{2}} \delta\left(\left(x-x_{0}\right)+\left(y-y_{0}\right) \tau\right)=\frac{1}{\sqrt{\left(x-x_{0}\right)^{2}+\left(y-y_{0}\right)^{2}}}
$$

We do not recover the exact solution which is $\delta\left(x-x_{0}\right) \delta\left(y-y_{0}\right)$, but a smooth peak at $\left(x_{0}, y_{0}\right)$ decaying as an inverse length for large distances. This approximate reconstruction method by back-projection is often used when an exact reconstruction formula is not available so that one has at least a rough idea of the expected result. More sophisticated back-projection methods have been subsequently developed under the name of "filtered back-projections" to improve the results with appropriate filters to be designed for each case. 


\section{The Radon transform on a half-upper-space}

\subsection{Generalities}

Here we consider a RT that integrates functions on lines in a half-upper-space, e.g. originating from a point on the $O x$-axis. It shall be designated by HRT, and serves as an intermediate between RT and VRT, as we shall see. It may be viewed also as a special class of X-ray transform in $\mathbb{R}^{2}$. In three dimensions, an X-ray transform integrates a function on a half-line, whose starting point lies on a curve. One possible notation for the X-ray transform in $\mathbb{R}^{3}$ is (see e.g. [28])

$$
\mathcal{D}_{a} f(\theta)=\int_{0}^{\infty} d t f(a+t \theta) \quad \theta \in \mathbb{S}^{2},
$$

where $a$ lies on a space curve and $\theta$ is a unit vector indicating a direction. Tuy has given the conditions on this curve under which this transform is invertible [29]. In two dimensions, it is often stated that its inversion is equivalent to the RT inversion. We shall give the explicit inversion formula which is very close to that of the RT, confirming this popular belief.

The HRT may arise in emission imaging as follows. A collimated X- or gamma ray detector is mounted on a vehicle describing a rectilinear trajectory. For each of its position, measurements of the radiation flux density entering the detector collimator shall be carried out for all possible angular directions.

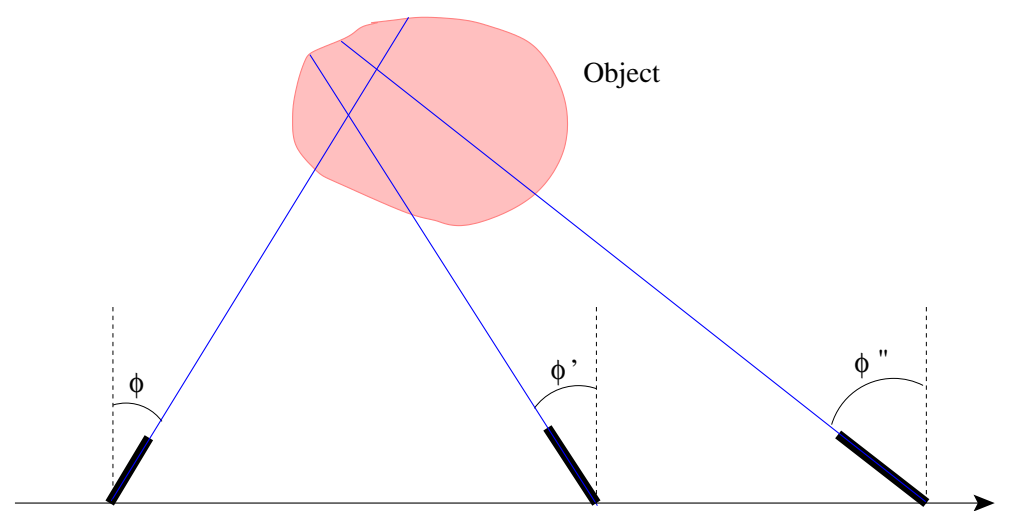

Figure 3. Half space emission scanning

Applications are

- at terrestrial level, to the imaging the radioactive distribution of a nuclear waste (or a nuclear reactor) with a swinging detector moving on a straight line path. The detector may be mounted on a robot or a drone.

- at galactic scale, to the imaging of gamma ray distribution of a galaxy by a swinging detector moving on a straight line trajectory. The detector may mounted on a spaceship. 


\subsection{Definition}

This transform, designated by $\mathcal{R}^{\natural}$, acts on functions defined on the upper half plane $\mathbb{E}=\left\{\mathbb{R} \times \mathbb{R}^{+}\right\}$(see Fig. 24) according to

$$
\mathcal{R}^{\natural} f(\xi, \tau)=\int_{0}^{\infty} d y \sqrt{1+\tau^{2}} f(\xi-y \tau, y) .
$$

It can be recast as classical Radon transform of $H(y) f(x, y)$ as

$$
\mathcal{R}^{\natural} f(\xi, \tau)=\int_{\mathbb{R}^{2}} d x d y \sqrt{1+\tau^{2}} \delta(x-\xi+y \tau) H(y) f(x, y),
$$

where $H(y)$ is the Heaviside unit step distribution.

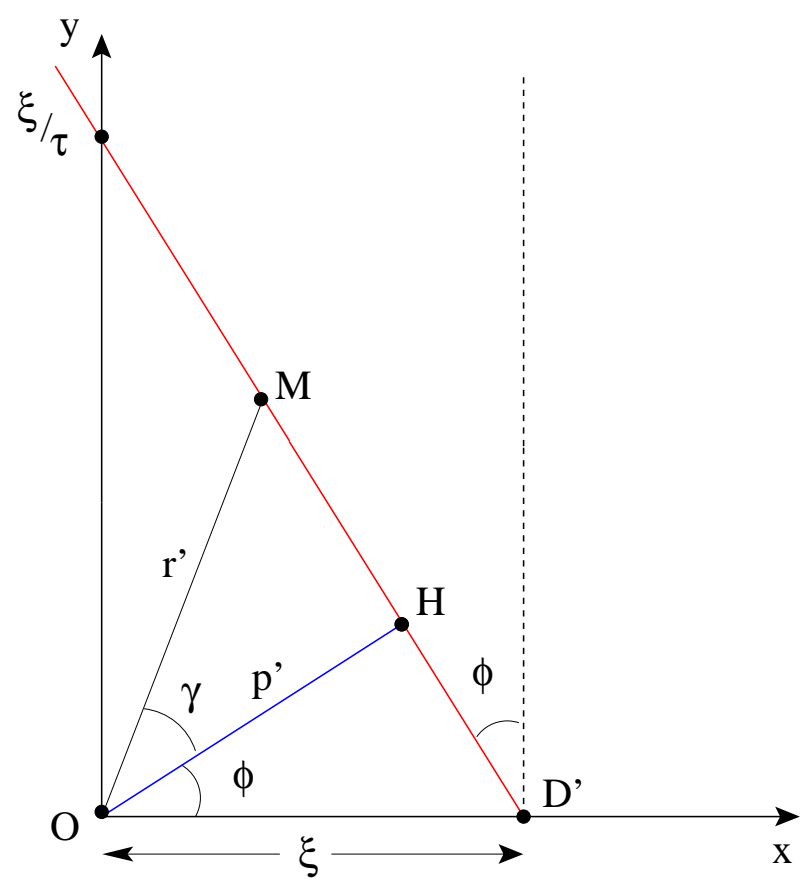

Figure 4. Half space Radon transform

Remark 3.1 Because of equation (82), many of the structural properties of $\mathcal{R}^{\natural}$, e.g. adjoint $\mathcal{R}^{\natural, \dagger}$, product $\mathcal{R}^{\natural} \mathcal{R}^{\natural, \dagger}$, back-projection can be immediately derived from those of the Radon transform $\mathcal{R}$.

\subsection{Inversion}

We shall see that the inversion procedure of the classical Radon transform can be applied and one gets not $f(x, y)$ but $H(y) f(x, y)$.

Theorem 3.2 The reconstruction formula for $\mathcal{R}^{\natural}$ is given by

$$
f(x, y)=\frac{1}{2 \pi^{2}} H(y) \int_{\mathbb{R}^{2}} \frac{d \tau d \xi}{\sqrt{1+\tau^{2}}} \frac{1}{(\xi-\tau y-x)} \frac{\partial}{\partial \xi} \mathcal{R}^{\natural} f(\xi, \tau)
$$


Proof: Using the Fourier representation $\tilde{f}(p, q)$ given by equation (13), and applying Fubini's theorem we have a new form

$$
\begin{aligned}
\frac{\mathcal{R}^{\natural} f(\xi, \tau)}{\sqrt{1+\tau^{2}}} & =\int_{\mathbb{R}^{2}} d p d q \tilde{f}(p, q) e^{2 i \pi p \xi} \int_{0}^{\infty} d y e^{-2 i \pi y(p \tau-q)} \\
& =\int_{\mathbb{R}^{2}} d p d q \tilde{f}(p, q) e^{2 i \pi p \xi} \frac{1}{2}\left[\delta(p \tau-q)+\frac{1}{i \pi} \frac{1}{p \tau-q}\right]
\end{aligned}
$$

By taking the Fourier transform in $\xi$ on both sides of this equation one has

$$
\int_{\mathbb{R}} d \xi e^{-2 i \pi \xi P} \frac{\mathcal{R}^{\natural} f(\xi, \tau)}{\sqrt{1+\tau^{2}}}=\frac{1}{2}\left[\tilde{f}(P, \tau P)+\frac{1}{i \pi} \int_{\mathbb{R}} d q \frac{\tilde{f}(P, q)}{p \tau-q}\right] .
$$

Now let

$$
\widetilde{f}(P, q)=\int_{\mathbb{R}} d \zeta e^{-2 i \pi \zeta q} g(P, \zeta)
$$

the second integral can be rewritten as (after applying Fubini's theorem)

$$
\begin{aligned}
\int_{\mathbb{R}} d q \frac{\tilde{f}(P, q)}{p \tau-q} & =-\int_{\mathbb{R}} d \zeta e^{-2 i \pi \zeta \tau P} g(P, \zeta) \int_{\mathbb{R}} d u \frac{e^{-2 i \pi \zeta u}}{u}, \\
& =-\int_{\mathbb{R}} d \zeta e^{-2 i \pi \zeta \tau P} g(P, \zeta)(-i \pi \operatorname{sgn} \zeta)
\end{aligned}
$$

where $\operatorname{sgn} \zeta$ is the sign function of $\zeta$. Since the first term in equation (27) is the integral

$$
\widetilde{f}(P, \tau P)=\int_{\mathbb{R}} d \zeta e^{-2 i \pi \zeta \tau P} g(P, \zeta),
$$

we obtain the result that

$$
\begin{aligned}
\int_{\mathbb{R}} d \xi e^{-2 i \pi \xi P} \frac{\mathcal{R}^{\natural} f(\xi, \tau)}{\sqrt{1+\tau^{2}}} & =\int_{\mathbb{R}} d \zeta e^{-2 i \pi \zeta \tau P} g(P, \zeta) \frac{1}{2}(1+\operatorname{sgn} \zeta) \\
& =\int_{0}^{\infty} d \zeta e^{-2 i \pi \zeta \tau P} g(P, \zeta) \\
& =\int_{\mathbb{R}} d \zeta e^{-2 i \pi \zeta \tau P} H(\zeta) g(P, \zeta) .
\end{aligned}
$$

Recall that $H(\zeta)$ is the Heaviside unit step distribution. For given $P \in \mathbb{R}$, we can multiply both sides of equation (28) by

$$
\int_{\mathbb{R}} d(\tau P) e^{2 i \pi \tau P z}
$$

to get

$$
\int_{\mathbb{R}} d(\tau P) e^{2 i \pi \tau P z} \int_{\mathbb{R}} d \xi e^{-2 i \pi \xi P} \frac{\mathcal{R}^{\natural} f(\xi, \tau)}{\sqrt{1+\tau^{2}}}=\int_{\mathbb{R}} d \zeta H(\zeta) g(P, \zeta) \delta(\zeta-z)=H(z) g(P, z) .
$$

Now, recalling that $\tau \in \mathbb{R}$ and $P \in \mathbb{R}$, we change variable

$$
d(\tau P)=|P| d \tau
$$

and extract for $P \in \mathbb{R}$ and for $z>0$

$$
g(P, z)=|P| \int_{\mathbb{R}^{2}} \frac{d \tau d \xi}{\sqrt{1+\tau^{2}}} e^{-2 i \pi(\xi-\tau z) P} \mathcal{R}^{\natural} f(\xi, \tau) .
$$


From equation (13), we get

$$
\widetilde{f}(P, q)=\int_{0}^{\infty} d z e^{-2 i \pi z q}|P| \int_{\mathbb{R}^{2}} \frac{d \tau d \xi}{\sqrt{1+\tau^{2}}} e^{-2 i \pi(\xi-\tau z) P} \mathcal{R}^{\natural} f(\xi, \tau) .
$$

Hence $f(x, y)$ can ne reconstructed as

$f(x, y)=\int_{\mathbb{R}^{2}} d p d q e^{2 i \pi(p x+q y)} \int_{0}^{\infty} d z e^{-2 i \pi z q}|p| \int_{\mathbb{R}^{2}} \frac{d \tau d \xi}{\sqrt{1+\tau^{2}}} e^{-2 i \pi(\xi-\tau z) p} \mathcal{R}^{\natural} f(\xi, \tau)$.

Permuting the integration order, we integrate first on $d q$ with $q \in \mathbb{R}$. This yields $\delta(z-y)$. The next integration is on $d z$ with the range $z \in \mathbb{R}^{+}$. This gives a non-zero result only if $y>0$, which is

$$
f(x, y)=H(y) \int_{\mathbb{R}^{2}} \frac{d \tau d \xi}{\sqrt{1+\tau^{2}}} \mathcal{R}^{\natural} f(\xi, \tau) \int_{\mathbb{R}}|p| d p e^{-2 i \pi p(\xi-\tau y-x)},
$$

(i.e. $y>0$ and $f(x, y)=0$ for $y<0$ ). Next from Fourier's table, one has

$$
\int_{\mathbb{R}}|p| d p e^{-2 i \pi p(\xi-\tau y-x)}=-\frac{1}{2 \pi^{2}} \frac{1}{(\xi-\tau y-x)^{2}} .
$$

Hence the final reconstruction formula is

$$
f(x, y)=-\frac{1}{2 \pi^{2}} H(y) \int_{\mathbb{R}^{2}} \frac{d \tau d \xi}{\sqrt{1+\tau^{2}}} \mathcal{R}^{\natural} f(\xi, \tau) \frac{1}{(\xi-\tau y-x)^{2}},
$$

where the Heaviside function describes the restriction on $y$. A partial integration on $d \xi$ removes the strong singularity at the pole yielding

$$
f(x, y)=\frac{1}{2 \pi^{2}} H(y) \int_{\mathbb{R}^{2}} \frac{d \tau d \xi}{\sqrt{1+\tau^{2}}} \frac{1}{(\xi-\tau y-x)} \frac{\partial}{\partial \xi} \mathcal{R}^{\natural} f(\xi, \tau)
$$

This result is identical to that of the line Radon transform but with a Heaviside function of $y$. This means that if we had only Radon data on the upper half plane we can only reconstruct what is in the upper half plane. One may venture to say that this is a limiting form of the standard support theorem for the line Radon transform, in which the circle limiting a ball in $\mathbb{R}^{2}$ reaches the limit of a line when its radius becomes infinite.

Remark 3.3 As the reconstruction formula is identical to that of the Radon transform except for the upper half plane limitation,

- the concept of back projection can be used also here provided that this restriction is respected,

- images of a point source, a curve of point source or a true two dimensional object have the same interpretations as in section 2.

\section{The Radon transform on V-lines}

Although the Radon transform on V-lines has been treated theoretically in [17], we reconsider it in a new formalism with Cartesian variables in this section. This transform may be viewed as the sum of two half-lines RT. 


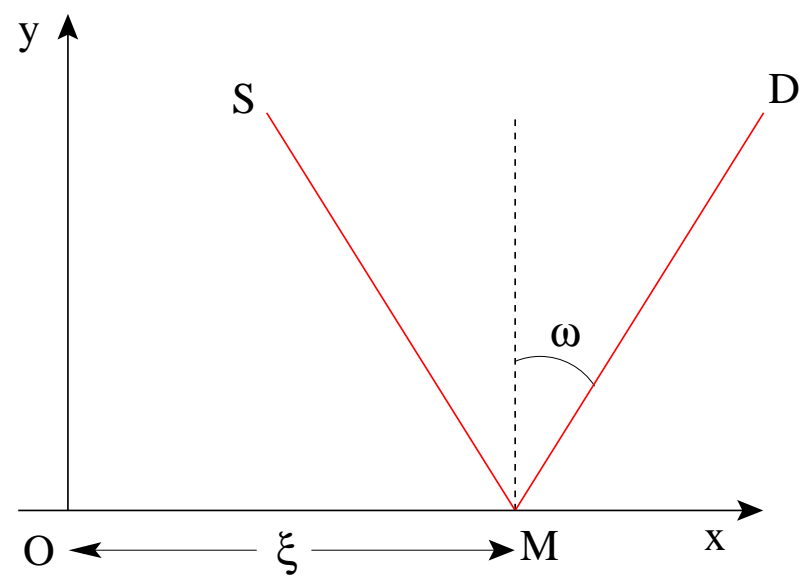

Figure 5. Set up for the V-line Radon transform

\subsection{Generalities}

Let $f(x, y)$ be an integrable function in $\mathbb{E}$. In this section, we are concerned with integrals of functions, not on lines of $\mathbb{R}^{2}$, but on $\mathrm{V}$-lines of equation

$$
|x-\xi|=y \tau,
$$

as depicted by Fig. 5. The half-lines $S M$ and $D M$ are parameterized by $O M=\xi$ and the angle $\omega \in[0, \pi / 2]$, or more conveniently by $\tan \omega=\tau$ with $\tau \in[0, \infty[$, so that each pair $(\xi, \tau) \in \mathbb{E}$ labels one such $\mathrm{V}$-lines.

Definition 4.1 We call the V-line Radon transform $\mathcal{R}_{V}$ of a function $f(x, y)$ the integral, whenever it exists

$$
\mathcal{R}_{V} f(\xi, \tau)=\int_{0}^{\infty} d r(f(\xi-\tau y, y)+f(\xi+\tau y, y)),
$$

where $d r=d y \sqrt{1+\tau^{2}}$ is the integration measure on the half-lines.

If conventional transmission tomography is based on rectilinear paths, the use of $\mathrm{V}$-line paths would allow a one-sided scanning procedure, which may be required in some situations. Forcing radiation along such V-line paths may be realized by a mirror. At present high quality mirrors exist for a broad range optical wavelengths including far infra-red or far ultraviolet lights, but perfectly reflecting mirrors for ionizing radiation are not available, since most reflecting devices for X-rays are limited by Bragg's diffraction and are inappropriate for use in transmission imaging. So V-line transmission imaging could work at the moment with laser radiation in object medium of intermediate optical thickness, such as in Single Scattering Optical Tomography, in which measurements of single scattered radiation along V-lines of fixed opening angles but moving rigidly in the plane. This type of V-lines has led to the concept of Radon transform on broken rays which is fully investigated by [19, 20, 21] as well as by [24] and by [22, 23]. This broken-ray transform may work in reflection modality with ionizing radiation when X-ray mirrors with Bragg reflection angles are used. 
As mentioned before, the V-line Radon transform may be used in emission imaging as already investigated by [15]. It has been shown that a bona fide twodimensional emission imaging system can be established [16]. Furthermore a Radon transform on V-lines of fixed axis direction and its extensions has been discussed in [17] for some emission imaging processes.

A few observations are in order:

- This definition is given for $\tau>0$, but by construction we have formally $\mathcal{R}_{V} f(\xi, \tau)=\mathcal{R}_{V} f(\xi,-\tau)$. Hence an extension to $\tau<0$ is possible with this property.

- Equation (40) is valid for integrable functions on $\mathbb{E}$.

- An alternative expression of the V-line RT as sum of two half-line RT is

$$
\mathcal{R}_{V} f(\xi, \tau)=\int_{\mathbb{R}^{2}} d x d y \sqrt{1+\tau^{2}}(\delta(x+y \tau-\xi)+\delta(x-y \tau-\xi)) H(y) f(x, y) .
$$

- Hence the kernel of $\mathcal{R}_{V}$ is the function $\mathcal{K}_{V}(\xi, \tau \mid x, y)$ on $\mathbb{E} \times \mathbb{E}$, given by

$$
\begin{aligned}
\mathcal{K}_{V}(\xi, \tau \mid x, y) & =\sqrt{1+\tau^{2}}[\delta(x-\xi+\tau y)+\delta(x-\xi-\tau y)] \\
& =\sqrt{1+\tau^{2}} \delta(|\xi-x|-y \tau) .
\end{aligned}
$$

- This kernel $\mathcal{K}_{V}(\xi, \tau \mid x, y) \in \mathbb{E} \times \mathbb{E}$, is the image of a point source (or PSF) put at site $(x, y)$, with the following symmetries

$$
\mathcal{K}_{V}(\xi, \tau \mid x, y)=\mathcal{K}_{V}(\xi,-\tau \mid x, y)=\mathcal{K}_{V}(\xi, \tau \mid x,-y)=\mathcal{K}_{V}(\xi,-\tau \mid x,-y) .
$$

In standard polar coordinates (see Fig. 6), the expression of the V-line Radon transform $\mathcal{R}_{V} f(s, \phi)$ of a function is

$$
\mathcal{R}_{V} f(s, \phi)=\int_{\frac{s}{\cos \phi}}^{\infty} \frac{d r}{\sqrt{1-\frac{s^{2}}{r^{2}}}}\left(f\left(r, \phi+\cos ^{-1} \frac{s}{r}\right)+f\left(r,-\phi+\cos ^{-1} \frac{s}{r}\right)\right),
$$

this expression will be used later in imaging applications.

\subsection{Adjoint V-line Radon Transform}

Definition 4.2 Let $\mathcal{R}_{V}^{\dagger}$ be the adjoint of $\mathcal{R}_{V}$. Its action on a integrable function $g(\xi, \tau)$ in $\mathbb{E}$ is given by

$$
\mathcal{R}_{V}^{\dagger} g(x, y)=\int_{0}^{\infty} \frac{d \tau}{1+\tau^{2}}(g(x+y \tau, \tau)+g(x-y \tau, \tau)) .
$$

To see how this form arises, we start from the V-line Radon transform kernel and integrate a function $g(\xi, \tau)$ with the measure of equation (4),

$\mathcal{R}_{V}^{\dagger} g(x, y)=\int_{0}^{\infty} \frac{d \tau}{\left(1+\tau^{2}\right)^{3 / 2}} \int_{-\infty}^{\infty} d \xi \sqrt{1+\tau^{2}}(\delta(x-\xi+\tau y)+\delta(x-\xi-\tau y)) g(\xi, \tau)$,

and integrate over $\xi$ to get equation (45).

It appears that $\mathcal{R}_{V}^{\dagger}$ and $\mathcal{R}_{V}$ are structurally alike and that

$$
\mathcal{R}_{V}^{\dagger} g(x, y)=\mathcal{R}_{V}^{\dagger} g(x,-y) .
$$




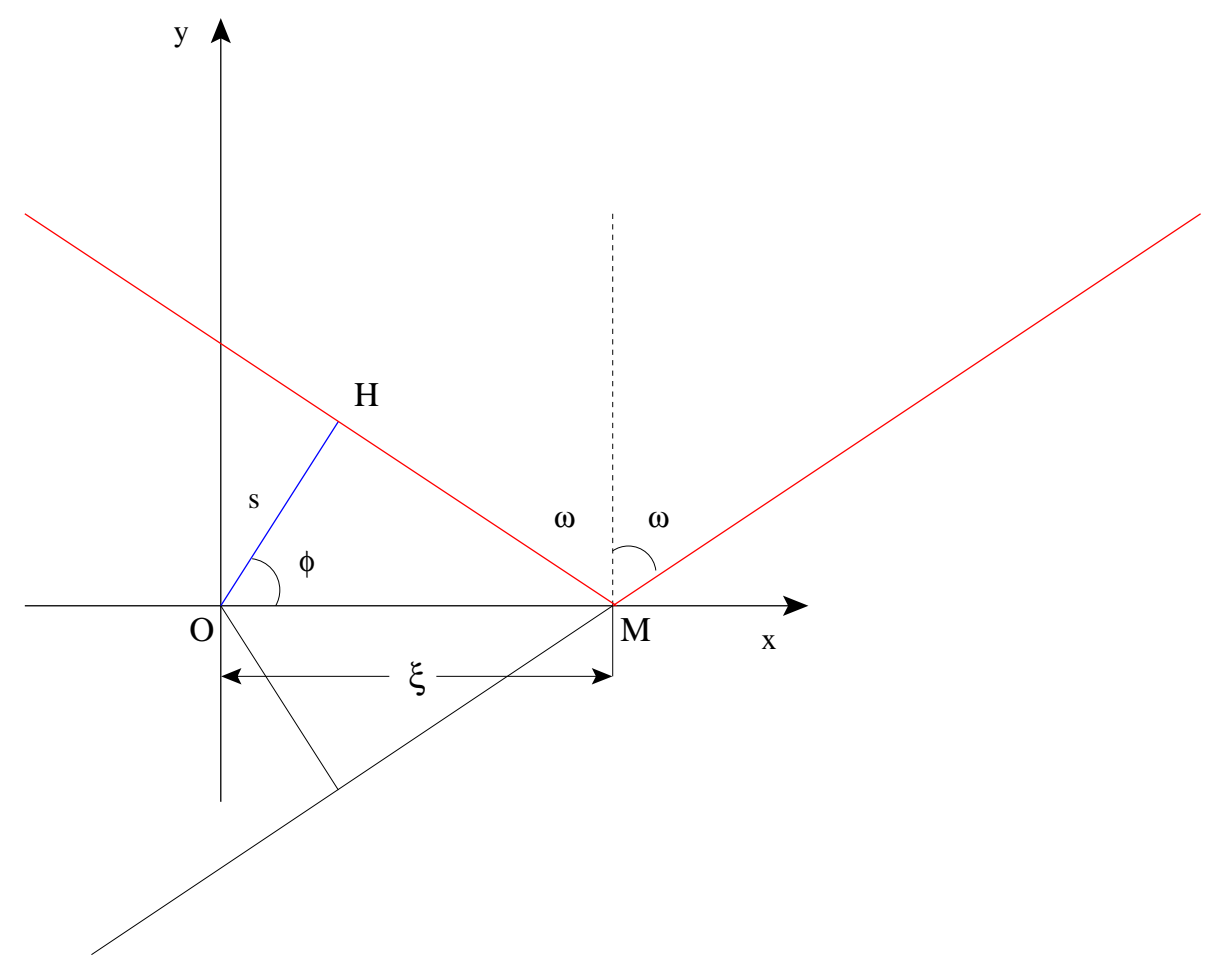

Figure 6. V-line Radon transform in classical Radon variables $(s, \phi)$ with $\phi=\omega$

\subsection{Product $\mathcal{R}_{V}^{\dagger} \mathcal{R}_{V}$}

Analogously to RT, the kernel of $\mathcal{R}_{V}^{\dagger} \mathcal{R}_{V}$ is computable and given by

Proposition 4.3 The action of the adjoint V-line Radon transform on the V-line PSF for a point source at $\left(x_{0}, y_{0}\right)$ is given by

$$
\mathcal{R}_{V}^{\dagger} \mathcal{K}\left(x, y \mid x_{0}, y_{0}\right)=\sum_{\epsilon= \pm} \frac{1}{\sqrt{\left(x-x_{0}\right)^{2}+\left(y+\epsilon y_{0}\right)^{2}}} .
$$

This formula shows only translational invariance in the $x$-direction as it should.

Proof. Using the definitions of the adjoint and the PSF of the V-line Radon transform, we have to evaluate the integral

$$
\begin{gathered}
\mathcal{R}_{V}^{\dagger} \mathcal{K}\left(x, y \mid x_{0}, y_{0}\right)=\int_{0}^{\infty} \frac{d \tau}{\left(1+\tau^{2}\right)^{3 / 2}}\left(1+\tau^{2}\right) \\
\times \int_{-\infty}^{\infty} d \xi[\delta(x-\xi+y \tau)+\delta(x-\xi-y \tau)]\left[\delta\left(x_{0}-\xi+y_{0} \tau\right)+\delta\left(x_{0}-\xi-y_{0} \tau\right)\right] .
\end{gathered}
$$

The $\xi$-integration can be easily carried out yielding

$$
\begin{gathered}
\mathcal{R}_{V}^{\dagger} \mathcal{K}\left(x, y \mid x_{0}, y_{0}\right)=\int_{0}^{\infty} \frac{d \tau}{\sqrt{1+\tau^{2}}} \\
{\left[\delta\left(\left(x_{0}-x\right)+\left(y_{0}-y\right) \tau\right)+\delta\left(\left(x_{0}-x\right)-\left(y_{0}-y\right) \tau\right)\right.}
\end{gathered}
$$




$$
\left.+\delta\left(\left(x_{0}-x\right)+\left(y_{0}+y\right) \tau\right)+\delta\left(\left(x_{0}-x\right)-\left(y_{0}+y\right) \tau\right)\right] .
$$

Now consider the relative positions of $(x, y)$ with respect to $\left(x_{0}, y_{0}\right)$ in the four quadrants:

$$
\left(x_{0}<x, y_{0}<y\right), \quad\left(x_{0}>x, y_{0}<y\right), \quad\left(x_{0}<x, y_{0}>y\right), \quad\left(x_{0}>x, y_{0}>y\right),
$$

we can integrate the delta function and obtain for all $(x, y)$ the result of equation (48).

This $\mathcal{R}_{V}^{\dagger}$-reconstruction of the V-line PSF reveals the point source at $\left(x_{0}, y_{0}\right)$. But although it is also a sharp peak at this point, this is not a rotational symmetric peak as in the case of the classical Radon transform. There is a contribution in the half-space $y>0$ due to the "image" point source at $\left(x_{0},-y_{0}\right)$.

Using this proposition 4.3, we have the following result

Corollary 4.4 We have

$$
\mathcal{R}_{V}^{\dagger}\left[\mathcal{R}_{V} f\right](x, y)=\int_{\mathbb{R}^{2}} d x_{0} d y_{0} f\left(x_{0}, y_{0}\right)\left\{\sum_{\epsilon= \pm} \frac{1}{\sqrt{\left(x-x_{0}\right)^{2}+\left(y+\epsilon y_{0}\right)^{2}}}\right\} .
$$

Proof. This result is obtained by using equation (45) and proposition (4.3). The right-hand-side appears as the sum of convolutions (instead of a simple convolution as it was the case of the classical Radon transform, see [9]) of $f(x, y)$ with the sum of two inverse distance potentials.

\subsection{Mapping to standard Radon Transform}

The V-line Radon transform may be recast a RT on a new family of functions $f_{e}(x, y)$, obtained by extending $f(x, y)$ to $\mathbb{R}^{2}$ via

$$
f_{e}(x, y)=\left\{\begin{array}{lll}
f(x, y) & \text { for } \quad y>0 \\
f(x,-y) & \text { for } \quad y<0
\end{array}\right.
$$

$f_{e}(x, y)$ may be expressed as $f_{e}(x, y)=H(y) f(x, y)+H(-y) f(x,-y)$, where $H(x)$ is the Heaviside unit step distribution and $f_{e}(x, y)=f_{e}(x,-y)$. We have

Proposition 4.5 We have the relation

$$
\mathcal{R}_{V} f(\xi, \tau)=\mathcal{R} f_{e}(\xi, \tau) .
$$

Proof. Put $y^{\prime}=-y$ in the second integral of equation (40) and regroup the terms after introducing the Heaviside function.

Remark 4.6 Note that one can put equally $\mathcal{R}_{V} f(\xi, \tau)$ under the form

$$
\mathcal{R}_{V} f(\xi, \tau)=\int_{\mathbb{R}^{2}} d x d y \sqrt{1+\tau^{2}} \delta(x-\xi-y \tau) f_{e}(x, y)
$$

\subsection{Inverse V-line Radon Transform}

A standard derivation in Cartesian formalism is given first followed by two other approaches based on the RT inversion formula and the integral form of the product $\mathcal{R}_{V}^{\dagger} \mathcal{R}_{V}$ 
4.5.1. Inversion in Cartesian coordinates For the convenience of the reader, we rederive the inversion formula in Cartesian coordinates.

Theorem 4.7 The inverse $V$-line transform $\mathcal{R}_{V}^{-1}$ is given by the reconstruction formula for $f(x, y)$, with $y>0$

$$
\begin{gathered}
f(x, y)= \\
-\frac{1}{2 \pi^{2}} \int_{0}^{\infty} \frac{d \tau}{\sqrt{1+\tau^{2}}} P . V . \int_{-\infty}^{\infty} d \xi\left(\frac{1}{\xi-x-y \tau}+\frac{1}{\xi-x+y \tau}\right) \frac{\partial}{\partial \xi} \mathcal{R}_{V} f(\xi, \tau) .
\end{gathered}
$$

Since formally (6.3) is invariant under $y \rightarrow(-y)$, on can write on the left-hand-side $f_{e}(x, y)=H(y) f(x, y)+H(-y) f(x,-y)$ instead of $f(x, y)$.

Proof. Because of translational invariance along the $O x$-axis, we introduce the partial Fourier representation of $f(x, y)$, with respect to the first variable, by

$$
f(x, y)=\int_{-\infty}^{\infty} d p e^{2 i \pi p x} \tilde{f}(p, y) .
$$

Then equation (5) reads

$$
\mathcal{R}_{V} f(\xi, \tau)=\sqrt{1+\tau^{2}} \int_{0}^{\infty} d y \int_{-\infty}^{\infty} d p e^{2 i \pi p \xi} 2 \cos 2 \pi p y \tau \tilde{f}(p, y) .
$$

The next step consists in applying the $\xi$-Fourier transform to equation (57)

$$
\int_{-\infty}^{\infty} d \xi e^{-2 i \pi \xi p} \mathcal{R}_{V} f(\xi, \tau)=\sqrt{1+\tau^{2}} \int_{0}^{\infty} d y 2 \cos 2 \pi p y \tau \tilde{f}(p, y)
$$

where $p \in \mathbb{R}$. The objective is to retrieve $\tilde{f}(p, y)$ from equation (58). This can be done by using the identity known in cosine Fourier transform $\ddagger$

$$
h(x)=2 \int_{0}^{\infty} d \zeta \cos 2 \pi \zeta x 2 \int_{0}^{\infty} d x^{\prime} \cos 2 \pi \zeta x^{\prime} h\left(x^{\prime}\right)
$$

valid for $x>0$.

Suppose first $p>0$, then we can multiply both sides of equation (58) by

$$
2 \int_{0}^{\infty} d(p \tau) \cos 2 \pi p \tau Y
$$

since $\tau>0$. Application of identity (59) yields

$$
\tilde{f}(p, Y)=2 \int_{0}^{\infty} d(p \tau) \cos 2 \pi p \tau Y \frac{1}{\sqrt{1+\tau^{2}}} \int_{-\infty}^{\infty} d \xi e^{-2 i \pi \xi p} \mathcal{R}_{V} f(\xi, \tau) .
$$

Suppose now that $p<0$, we put $p=-p^{\prime}$ with $p^{\prime}>0$, then going through the same procedure we have

$$
\widetilde{f}\left(-p^{\prime}, Y\right)=2 \int_{0}^{\infty} d\left(p^{\prime} \tau\right) \cos 2 \pi p^{\prime} \tau Y \frac{1}{\sqrt{1+\tau^{2}}} \int_{-\infty}^{\infty} d \xi e^{2 i \pi \xi p^{\prime}} \mathcal{R}_{V} f(\xi, \tau) .
$$

These two cases may be expressed by the following single formula

$$
\widetilde{f}(p, Y)=2|p| \int_{0}^{\infty} \frac{d \tau}{\sqrt{1+\tau^{2}}} \cos 2 \pi|p| \tau Y \int_{-\infty}^{\infty} d \xi e^{-2 i \pi \xi p} \mathcal{R}_{V} f(\xi, \tau) .
$$

$\ddagger$ A similar identity exists under the name of Hankel identity for Bessel functions of the first kind. 


$$
\begin{aligned}
& f(x, y) \text { is recovered via equation (56) as } \\
& f(x, y)=\int_{-\infty}^{\infty} d p e^{2 i \pi p x} 2|p| \int_{0}^{\infty} \frac{d \tau}{\sqrt{1+\tau^{2}}} \cos 2 \pi|p| \tau y \int_{-\infty}^{\infty} d \xi e^{-2 i \pi \xi p} \mathcal{R}_{V} f(\xi, \tau)
\end{aligned}
$$

Using Fubini's theorem to reshuffle the integration order, we integrate first on $p$

$$
\begin{array}{r}
\int_{-\infty}^{\infty} d p|p|\left(e^{2 i \pi p(x-\xi+y \tau)}+e^{2 i \pi p(x-\xi-y \tau)}\right)= \\
\int_{-\infty}^{\infty} \frac{d z}{2 \pi^{2}}\left(\delta^{\prime}(\xi-x-y \tau-z)+\delta^{\prime}(\xi-x+y \tau-z)\right) .
\end{array}
$$

Putting this result (64) in equation (63), we obtain

$$
\begin{gathered}
f(x, y)=-\frac{1}{2 \pi^{2}} \int_{0}^{\infty} \frac{d \tau}{\sqrt{1+\tau^{2}}} \\
\text { P.V. } \int_{-\infty}^{\infty} \frac{d z}{z}\left(\mathcal{R}^{\prime}{ }_{V} f(x+y \tau+z, \tau)+\mathcal{R}^{\prime}{ }_{V} f(x-y \tau+z, \tau)\right),
\end{gathered}
$$

and

$$
\mathcal{R}_{V}^{\prime} f(x, y)=\frac{\partial}{\partial x} \mathcal{R}_{V} f(x, y) .
$$

By a simple change of variable, we can put the result under the usual form of [17], given by equation (6.3).

So far we have kept $y>0$, hence the result of equation (65) holds for $y>0$. But the expression of equation (65) remains the same if one changes $y$ to $-y$. Consequently one should write the left-hand-side of equation (65) as $H(y) f(x, y)+H(-y) f(x, y)$, so that it is valid for all $y \in \mathbb{R}$.

Remark 4.8 Of course formula (65) can be viewed as a back-projection operation according to equation (74) with filtered data

$$
g(x \pm y \tau)=-\frac{1}{2 \pi^{2}} \frac{1}{\sqrt{1+\tau^{2}}} \text { P.V. } \int_{-\infty}^{\infty} \frac{d z}{z}\left(\frac{\partial}{\partial \xi} \mathcal{R}_{V} f(x \pm y \tau+z, \tau)\right) .
$$

4.5.2. Inversion via the inverse of the Radon transform As pointed out in proposition $4.5, \mathcal{R}_{V}$ on $f(x, y)$ may be converted into $\mathcal{R}$ on functions $f_{e}(x, y)$. So one may use the $\mathcal{R}$ inversion formula to obtain the inversion formula for $\mathcal{R}_{V} \S$.

Proposition 4.9 The inversion of the V-line Radon transform via the classical inverse Radon transform leads to the same reconstruction formula.

Proof. Start from the classical Radon transform inverse formula with the data $\mathcal{R}_{V} f(\xi, \tau)$

$$
f_{e}(x, y)=-\frac{1}{2 \pi^{2}} \int_{-\infty}^{\infty} \frac{d \tau}{\sqrt{1+\tau^{2}}} \text { P.V. } \int_{-\infty}^{\infty} d \xi \frac{1}{(\xi-x-y \tau)} \frac{\partial}{\partial \xi} \mathcal{R}_{V} f(\xi, \tau)
$$

Split the $\tau$-integration range into $]-\infty, 0] \cup[0, \infty[$. In the second integral over the range $]-\infty, 0]$, put $\tau=-\tau^{\prime}$ and express it as

$$
-\frac{1}{2 \pi^{2}} \int_{0}^{\infty} ? ? d \tau^{\prime} \frac{1}{\sqrt{1+\tau^{\prime 2}}} \text { P.V. } \int_{-\infty}^{\infty} d \xi \frac{1}{\xi-x+y \tau^{\prime}} \frac{\partial}{\partial \xi} \mathcal{R}_{V} f\left(\xi,-\tau^{\prime}\right) .
$$

Since $\mathcal{R}_{V} f(\xi, \tau)=\mathcal{R}_{V} f(\xi,-\tau)$, we reproduce the result of theorem (4.7).

$\S$ This point was touched upon in [13], but not completely developed. 
4.5.3. An alternative inversion formula The solution of this convolution integral equation leads to a new inversion formula for the V-line Radon transform data

$$
g(\xi, \tau)=\mathcal{R}_{V} f(\xi, \tau) .
$$

Recalling the Fourier representation of the convolution product (equation (51)), we may write

$$
\mathcal{R}_{V} g(x, y)=\int_{-\infty}^{\infty} d p \int_{-\infty}^{\infty} d q e^{2 i \pi p x} 2 \cos 2 i \pi q y \frac{\tilde{f}(p, q)}{\sqrt{p^{2}+q^{2}}}
$$

since the two-dimensional Fourier transform of $1 / \sqrt{x^{2}+y^{2}}$ is $1 / \sqrt{p^{2}+q^{2}}$. Consequently

$$
\widetilde{f}(p, q)=\sqrt{p^{2}+q^{2}} \int_{0}^{\infty} d y 2 \cos 2 i \pi q y \int_{-\infty}^{\infty} d x e^{-2 i \pi p x} \mathcal{R}_{V} g(x, y)
$$

The function $f(x, y)$ is then recovered from its Fourier components $\widetilde{f}(p, q)$

$$
f(x, y)=\int_{-\infty}^{\infty} \int_{-\infty}^{\infty} d p d q e^{2 i \pi(p x+q y)} \tilde{f}(p, q) .
$$

This result generalizes the one known for the classical Radon transform [9]. In practice, the filter $\sqrt{p^{2}+q^{2}}$ must also be apodized in Fourier space $(p, q)$.

Remark 4.10 What was said about the image of a point source, of a series of point sources on a curve and of a true two-dimensional object may be repeated here under two conditions

- restriction to the upper part of the plane with $H(y)$,

- from equation (42), one can see that the image of a unit point source at $\left(x_{0}, y_{0}\right)$ has a support which is a $V$-line in $(\xi, \tau)$ space of equation $\left|\xi-x_{0}\right|-y_{0} \tau=0$. Lines in Radon space are "optically" reflected on the Ox-axis, as if this axis is a mirror.

\subsection{Back-projection of V-line Radon transform data}

How should the concept of back-projection be extended to the V-line transform? Let a projection (the measurement of radiation intensity after its travel through a V-line) be called $g(\xi, \tau)$. By back-projecting this data on a $\mathrm{V}$-line, we mean finding a function of $(x, y) \in \mathbb{R}^{2}$, which should take a constant value on the $\mathrm{V}$-line with vertex at abscissa $\xi$ and slope $\tau$. Following the same reasoning as in the classical Radon transform we can see that the function

$$
H(x-\xi) g(x-y \tau, \tau)+H(\xi-x) g(x+y \tau, \tau),
$$

where $H(x)$ is the Heaviside unit-step function, takes the value $g(\xi, \tau)$ on the V-line of parameters $(\xi, \tau)$.

However there is a marked difference with the previous case. If we seek to determine the value of the function representing the back-projection of the data $g(\xi, \tau)$ at a point $(x, y) \in \mathbb{R}^{2}$ for fixed $\tau$, we see that this point stands at the intersection of two $\mathrm{V}$-lines with the same $\tau$ but with two different abscissas $\xi_{-}$and $\xi_{+}$, given in terms of $(x, y)$ and $\tau$ by

$$
\xi_{-}=(x-y \tau), \quad \text { and } \quad \xi_{+}=(x+y \tau) .
$$




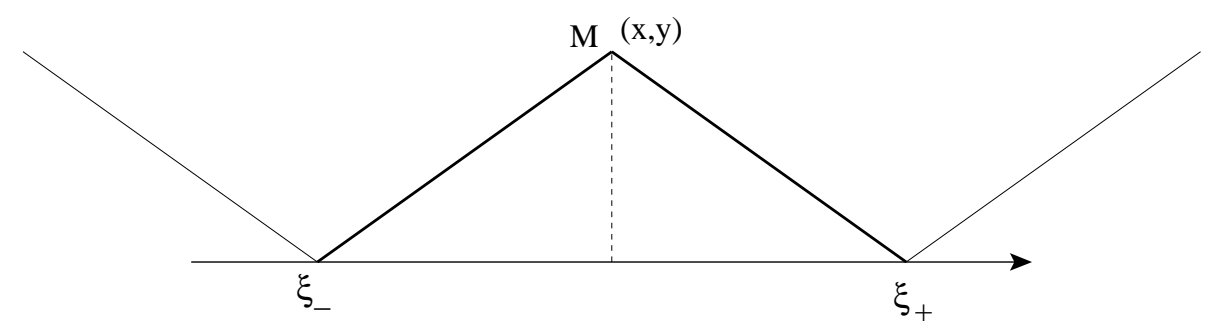

Figure 7. Contributions of two V-lines at one point $(x, y) \in \mathbb{R}^{2}$

This situation is clearly described by Fig. 7

Hence the back-projected radiation intensity at $(x, y) \in \mathbb{R}^{2}$ for given $\tau$ is the sum $g\left(\xi_{-}, \tau\right)+g\left(\xi_{+}, \tau\right)$. Consequently the sought V-line back-projection function of the data $g(\xi, \tau)$ must be the sum $g(x-y \tau, \tau)+g(x+y \tau, \tau)$. Image reconstruction by this simple back-projection is then completed by an integration on $\tau$, i.e.

$$
f(x, y) \approx \int_{0}^{\infty} d \tau(g(x-y \tau, \tau)+g(x+y \tau, \tau)) .
$$

Of course, as expected this unsophisticated image reconstruction is plagued by defects, which should be removed by filtered back-projection. In this respect the action of $\mathcal{R}_{V}^{\dagger}$ on a projection $g(\xi, \tau)$ may be interpreted as some kind of filtered back projection in which the filter is $1 / 1+\tau^{2}$.

\section{Geometric inversion and effects on Radon transforms}

Geometric inversion is an involutive point transformation which maps $(x, y) \in \mathbb{R}^{2}$ onto $\left(x^{\prime}, y^{\prime}\right) \in \mathbb{R}^{2}$, such that

$$
(x, y) \rightarrow\left(x^{\prime}, y^{\prime}\right) \frac{R^{2}}{x^{2}+y^{2}}(x, y),
$$

where $R$ is the radius of the inversion circle centered at the coordinate origin $O$.

This transformation converts a straight line into a circle intersecting $O$ as follows. Let the straight line equation be $x^{\prime} \pm y^{\prime} \tau-\xi=0$. By inserting relations (75) into this line equation, we obtain the equation of a circle intersecting the origin

$$
x^{2}+y^{2}-\xi^{\prime} x \pm \tau \xi^{\prime} y=0,
$$

where $\xi^{\prime}=R^{2} / \xi$. The radius of the circle is $\xi^{\prime} \sqrt{1+\tau^{2}} / 2$ and its center is at site $\left(\xi^{\prime} / 2, \tau \xi^{\prime} / 2\right)$.

For clarity, functions that are integrated on straight line paths are designated by $f(x, y)$ and functions integrated on circular paths shall be designated by $g\left(x^{\prime}, y^{\prime}\right)$. Their physical significance will be specified later in applications.

\subsection{Effect on $\mathcal{R}, \mathcal{R}^{\natural}$ and $\mathcal{R}_{V}$}

We now look at the effect of geometric inversion on the full line, half-line and V-line Radon Transforms. It turns them into Radon Transforms on a full circle, a circular 
arc and a pair of "supplementary" circular arcs intersecting $O$. To see this, we need the Jacobian of the inversion

$$
d x^{\prime} d y^{\prime}=\left|\frac{\partial\left(x^{\prime}, y^{\prime}\right)}{\partial(x, y)}\right| d x d y=\frac{R^{4}}{\left(x^{2}+y^{2}\right)^{2}} d x d y
$$

as well as the "sifting" property of the delta function, see [9].

- The defining equation of the full line Radon Transform

is now converted into

$$
\mathcal{R} f(\xi, \tau)=\sqrt{1+\tau^{2}} \int_{\mathbb{R}^{2}} d x^{\prime} d y^{\prime} \delta\left(x^{\prime}-y^{\prime} \tau-\xi\right) f(x, y) .
$$

$$
\mathcal{R} f(\xi, \tau)=\xi^{\prime} \sqrt{1+\tau^{2}} \int_{\mathbb{R}^{2}} d x d y \delta\left(x^{2}+y^{2}-\xi^{\prime} x-\xi^{\prime} \tau y\right) g_{R}(x, y),
$$

which is the integral of a new function $g_{R}(x, y)$

$$
g_{R}(x, y)=\frac{R^{2}}{x^{2}+y^{2}} f\left(\frac{R^{2} x}{x^{2}+y^{2}}, \frac{R^{2} y}{x^{2}+y^{2}}\right) .
$$

on a full circle intersecting the origin $O$ of radius $\xi^{\prime} \sqrt{1+\tau^{2}} / 2$ and with center at site $\left(\xi^{\prime} / 2, \tau \xi^{\prime} / 2\right)$. For all $g_{R}(x, y)$, obtainable from a given $f(x, y)$, this equation expresses the definition of the Radon transform on circles through the origin $O$, coined by A M Cormack as circular Radon Transform $\mathcal{R}_{C}$. Hence we have

$$
\mathcal{R} f(\xi, \tau)=\mathcal{R}_{C} g_{R}\left(\xi^{\prime}, \tau\right) .
$$

- Similarly, the defining equation of the half-line Radon Transform

$$
\mathcal{R}^{\natural} f(\xi, \tau)=\int_{\mathbb{R}^{2}} d x d y \sqrt{1+\tau^{2}} \delta(x-\xi+y \tau) H(y) f(x, y),
$$

is, following the same approach, now converted into

$$
\mathcal{R}^{\natural} f(\xi, \tau)=\xi^{\prime} \sqrt{1+\tau^{2}} \int_{\mathbb{R}^{2}} d x d y \delta\left(x^{2}+y^{2}-\xi^{\prime} x-\xi^{\prime} \tau y\right) H(y) g_{R}(x, y),
$$

the integral of $g_{R}(x, y)$ on the circular arc of a circle of radius $\xi^{\prime} \sqrt{1+\tau^{2}} / 2$ intersecting the origin $O$ and located in the upper $y>0$ part of the plane. Here we use the sign ${ }^{\natural}$ to represent the restriction to the upper half plane

$$
\mathcal{R}^{\natural} f(\xi, \tau)=\mathcal{R}_{C}^{\natural} g_{R}\left(\xi^{\prime}, \tau\right) .
$$

- Finally, the defining equation of the V-line Radon Transform

$$
\mathcal{R}_{V} f(\xi, \tau)=\int_{\mathbb{R}^{2}} d x d y \sqrt{1+\tau^{2}}(\delta(x+y \tau-\xi)+\delta(x-y \tau-\xi)) H(y) f(x, y)
$$

shall be transformed into

$$
\begin{gathered}
\mathcal{R}_{V} f(\xi, \tau)= \\
\xi^{\prime} \sqrt{1+\tau^{2}} \int_{\mathbb{R}^{2}} d x d y\left(\delta\left(x^{2}+y^{2}-\xi^{\prime} x-\xi^{\prime} \tau y\right)+\delta\left(x^{2}+y^{2}-\xi^{\prime} x+\xi^{\prime} \tau y\right)\right) H(y) g_{R}(x, y),
\end{gathered}
$$

the integral of $g_{R}(x, y)$ on two circular arcs of a circle of radius $\xi^{\prime} \sqrt{1+\tau^{2}} / 2$ intersecting the origin $O$ and with centers located respectively at $\left(\xi^{\prime} / 2, \pm \tau \xi^{\prime} / 2\right)$ in the upper $y>0$ part of the plane. The right-hand-side of equation (86) represents the Radon transform on a pair of circular arcs in the upper half plane parameterized by $\pm \tau \|$, which shall be denoted by $\mathcal{R}_{C C}^{\natural}$. Hence, we have

$$
\mathcal{R}_{V} f(\xi, \tau)=\mathcal{R}_{C C}^{\natural} g_{R}\left(\xi^{\prime}, \tau\right) .
$$

\| They subtend supplementary inscribed angles and shall be called supplementary circular arcs. 
Consequently, these Radon transforms $\left(\mathcal{R}_{C}, \mathcal{R}_{C}^{\natural}, \mathcal{R}_{C C}^{\natural}\right)$ are directly related successively to $\left(\mathcal{R}, \mathcal{R}^{\natural}, \mathcal{R}_{V}\right)$; properties of one group may be deduced from those of the other group and vice versa.

\subsection{Reverse effect on $\mathcal{R}_{C}, \mathcal{R}_{C}^{\natural}$ and $\mathcal{R}_{C C}^{\natural}$}

The involutive nature of geometric inversion induces the reverse passage as follows.

- The defining equation for Cormack circular Radon Transform, i.e. Radon Transform on full circles passing through the coordinate origin $O$ is

$$
\mathcal{R}_{C} g\left(\xi^{\prime}, \tau\right)=\xi^{\prime} \sqrt{1+\tau^{2}} \int_{\mathbb{R}^{2}} d x^{\prime} d y^{\prime} \delta\left(x^{\prime 2}+y^{\prime 2}-\xi^{\prime} x^{\prime}-\tau \xi^{\prime} y^{\prime}\right) g\left(x^{\prime}, y^{\prime}\right)
$$

Under geometric inversion, it turns into

$$
\mathcal{R}_{C} g\left(\xi^{\prime}, \tau\right)=\sqrt{1+\tau^{2}} \int_{\mathbb{R}^{2}} d x d y \delta(x+\tau y-\xi) f_{R}(x, y)
$$

with

$$
f_{R}(x, y)=\frac{R^{2}}{x^{2}+y^{2}} g\left(\frac{R^{2} x}{x^{2}+y^{2}}, \frac{R^{2} y}{x^{2}+y^{2}}\right) .
$$

Thus we can write

$$
\mathcal{R}_{C} g\left(\xi^{\prime}, \tau\right)=\mathcal{R} f_{R}(\xi, \tau) .
$$

- The upper half plane circular arc Radon Transform of $g\left(x^{\prime}, y^{\prime}\right)$

$$
\mathcal{R}_{C}^{\natural} g\left(\xi^{\prime}, \tau\right)=\xi^{\prime} \sqrt{1+\tau^{2}} \int_{\mathbb{R}^{2}} d x^{\prime} d y^{\prime} \delta\left(x^{\prime 2}+y^{\prime 2}-\xi^{\prime} x^{\prime}-\tau \xi^{\prime} y^{\prime}\right) H\left(y^{\prime}\right) g\left(x^{\prime}, y^{\prime}\right),
$$

becomes under geometric inversion

$$
\mathcal{R}_{C}^{\natural} g\left(\xi^{\prime}, \tau\right)=\sqrt{1+\tau^{2}} \int_{\mathbb{R}^{2}} d x d y \delta(x+\tau y-\xi) H(y) f_{R}(x, y)
$$

Hence one has

$$
\mathcal{R}_{C}^{\natural} g\left(\xi^{\prime}, \tau\right)=\mathcal{R}^{\natural} f_{R}(\xi, \tau) .
$$

- Finally the upper half plane double supplementary circular arcs Radon Transform of $g\left(x^{\prime}, y^{\prime}\right)$ is

$$
\begin{gathered}
\mathcal{R}_{C C}^{\natural} g\left(\xi^{\prime}, \tau\right)=\xi^{\prime} \sqrt{1+\tau^{2}} \times \\
\int_{\mathbb{R}^{2}} d x^{\prime} d y^{\prime}\left(\delta\left(x^{\prime 2}+y^{\prime 2}-\xi^{\prime} x^{\prime}-\tau \xi^{\prime} y^{\prime}\right)+\delta\left(x^{\prime 2}+y^{\prime 2}-\xi^{\prime} x^{\prime}+\tau \xi^{\prime} y^{\prime}\right)\right) H\left(y^{\prime}\right) g\left(x^{\prime}, y^{\prime}\right),
\end{gathered}
$$

is converted by geometric inversion into the V-line Radon Transform

$$
\mathcal{R}_{C C}^{\natural} g_{R}\left(\xi^{\prime}, \tau\right)=\sqrt{1+\tau^{2}} \int_{\mathbb{R}^{2}} d x d y(\delta(x+\tau y-\xi)+\delta(x-\tau y-\xi)) H(y) f_{R}(x, y),(96)
$$

or

$$
\mathcal{R}_{C C}^{\natural} g\left(\xi^{\prime}, \tau\right)=\mathcal{R}_{V} f_{R}(\xi, \tau)
$$




\section{Application to Norton's modality of Compton Scatter Tomography (CST)}

\subsection{Compton scatter tomography}

In the search for more efficient radiation imaging processes, the idea of Compton scatter tomography (CST) has appeared as early as 1974 [35]. It consists of obtaining, instead of the usual attenuation map in transmission imaging or the radioactivity density of a radio-tracer in emission imaging, the electric charge density map in a two-dimensional slice of an object via the Compton effect. At first, the value of this electric charge density is obtained point by point then line by line [33, 35]. A major step was achieved when the use of wide angle source and detector was proposed to vastly improve the sensitivity [36]. In this way the amount of radiation at a given scattered energy registered by the detector comes from the set of scattering sites situated on a circular arc connecting the emitting radiation source to the point detector subtending an angle $(\pi-\omega)$, where $\omega$ is the Compton scattering angle corresponding to the detected energy $E(\omega)$. But this quantity is essentially proportional to the integral of the object electronic charge density along an isogonic circular arc, as shown in Fig. 8. This is how the concept of Radon transform on circular arcs came up in Compton scatter tomography.

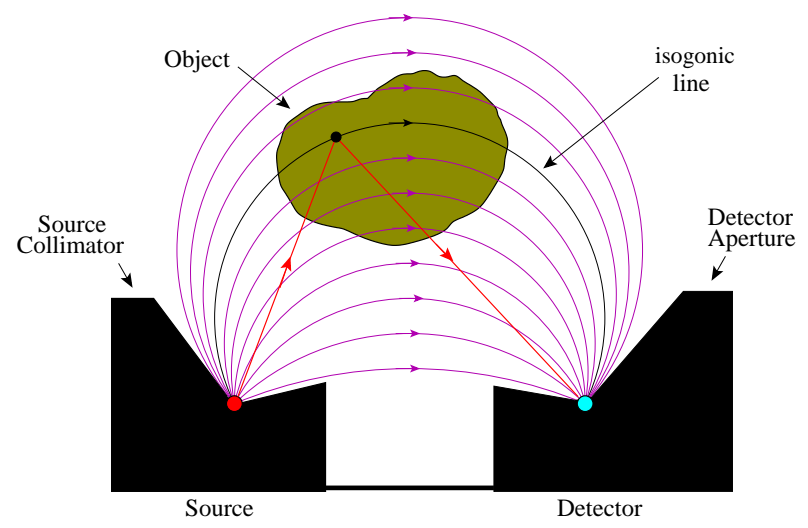

Figure 8. Circular Arcs in Compton Scatter Tomography, see [41]

\subsection{S J Norton Compton scatter tomography}

It turns out that there are many possible classes of such circular arcs, each one may correspond to a specific class of Radon transform. Here, we are only concerned with the class of circular arcs which start from a fixed radiation point source $S$ and which end at a detecting point $D$ moving on a straight line originating from $S$. In 1995 elaborating on previous works, such as [37], S J Norton has made use of this class of circular arcs to establish the first operative modality of Compton scatter tomography in [34] . However to reconstruct the electric charge density, he proposed to use the inverse formula of the Radon Transform on full isogonic circles passing through the coordinate

ๆ Other classes of circular arcs leading to other Compton scatter tomography modalities have been discussed in $[38,39,40,41]$. 
origin $S$, which is also the external radiation source. This prescription means that the detector collects indifferently scattered radiation at scattering angles $\omega$ or $(\pi-\omega)$. But physically, the detector can only register selectively only scattered radiation flux density either at $\omega$ or at $(\pi-\omega)$. If $\omega$ is chosen, then it is the $\mathcal{R}_{C}^{\natural}$, which should be used. Let $g\left(x^{\prime}, y^{\prime}\right)$ be the sought electric charge density. The data is then represented by $\mathcal{R}_{C}^{\natural} g\left(\xi^{\prime}, \tau\right)$. But equation (94) shows that $\mathcal{R}_{C}^{\natural} g\left(\xi^{\prime}, \tau\right)=\mathcal{R}^{\natural} f_{R}(\xi, \tau)$, where $f_{R}(x, y)$ of equation (90) represents an apparent density (apparent attenuation map or apparent radio-active source density), subjected to a half-line Radon Transform, see Fig 9. Thus from equation (38) we can write down the reconstruction formula for $f_{R}(x, y)$ as

$$
f_{R}(x, y)=\frac{1}{2 \pi^{2}} H(y) \text { P.V. } \int_{\mathbb{R}^{2}} \frac{d \tau d \xi}{\sqrt{1+\tau^{2}}} \frac{1}{\xi-\tau y-x} \frac{\partial}{\partial \xi} \mathcal{R}_{C}^{\natural} g\left(\frac{R^{2}}{\xi}, \tau\right) .
$$

The last step consists in going back to the original electric charge density by

$$
g\left(x^{\prime}, y^{\prime}\right)=\frac{R^{2}}{x^{\prime 2}+y^{\prime 2}} f_{R}\left(\frac{R^{2} x^{\prime}}{x^{\prime 2}+y^{\prime 2}}, \frac{R^{2} y^{\prime}}{x^{\prime 2}+y^{\prime 2}}\right) .
$$

In Fig.9, we have represented the Norton's modality for Compton scatter tomography (CST). The monochromatic radiation source is at $S$, the mobile detecting point $D$ is on the $O x$-axis and register the scattered radiation flux density under the Compton scattering angle $(\pi-\omega)$. $\Gamma$ is the inversion circle of radius $R$ and center $S$. The original object is "scanned" by the set of upper circular arcs, corresponding to a Radon transform on these circular arcs. Under inversion, it is transformed into an apparent (or inverse) objet "scanned" by half-lines corresponding to a Radon transform on these half-lines. The circular arc $S M D$ is transformed into the half-line $D^{\prime} M^{\prime}$ and $S D \cdot S D^{\prime}=R^{2}$. Image reconstruction is done by inversion of the half-line Radon transform and restoration of the initial object function with the help of equation (99). 


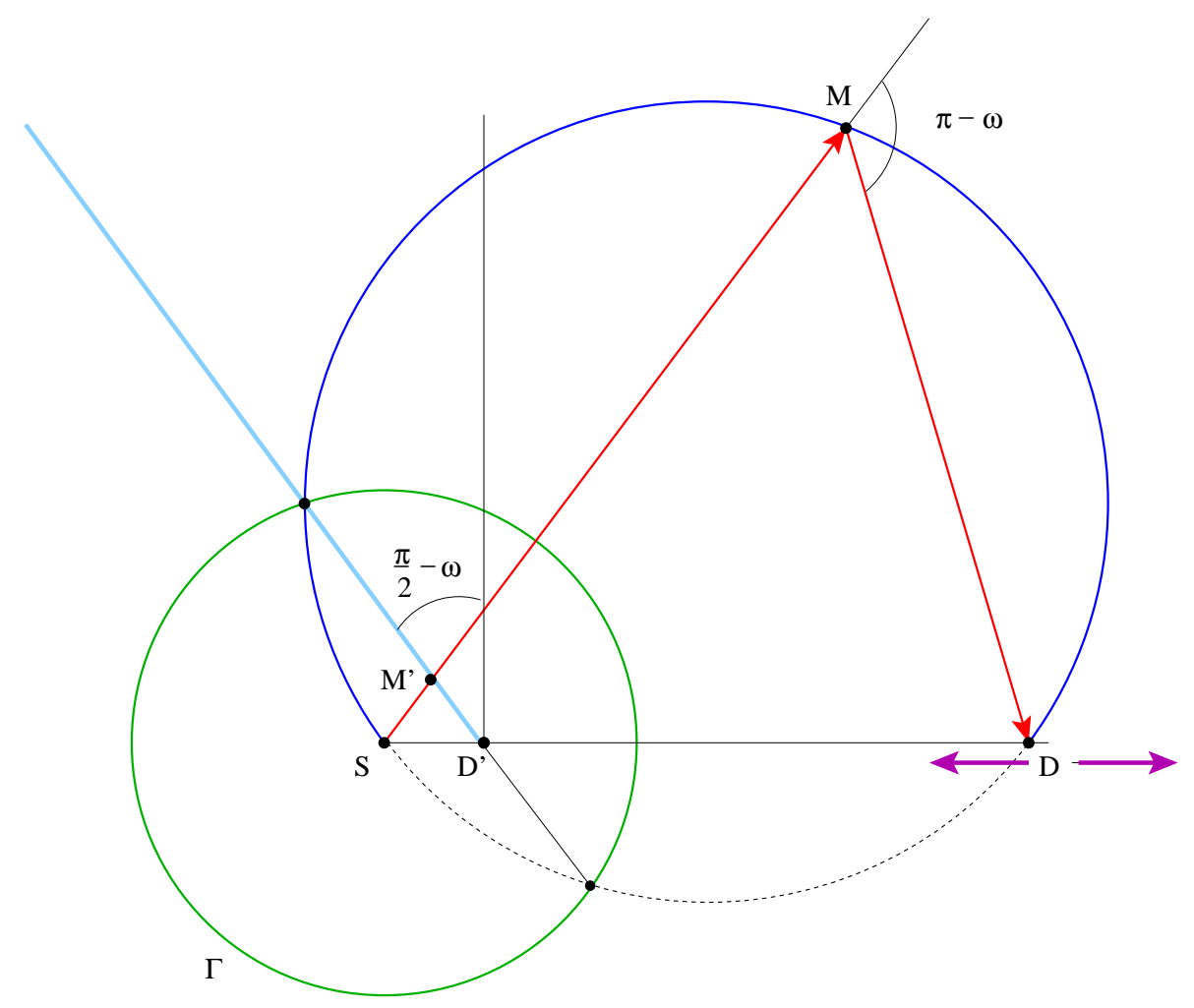

Figure 9. Scanning in Norton CST - mapping to half-line scanning. Here the Compton scattering angle is $(\pi-\omega)$

\subsection{A new modality for Norton's Compton scatter tomography arising from the} $V$-line Radon transform

We consider now a new modality of Norton's Compton Tomography (CST) in which the detector $D$ registers the sum of two successive measurements of scattered radiation at energies $E(\omega)$ and $E(\pi-\omega)$, as shown in figure below. This sum is due to the integration of the electric charge density $g\left(x^{\prime}, y^{\prime}\right)$ on two supplementary circular arcs in the upper half part of the plane. Therefore this data is correctly represented by $\mathcal{R}_{C C}^{\natural} g\left(\xi^{\prime}, \tau\right)$, see Fig 10 . But equation (97) shows that $\mathcal{R}_{C C}^{\natural} g\left(\xi^{\prime}, \tau\right)=\mathcal{R}_{V} f_{R}(\xi, \tau)$. The reconstruction formula for $f_{R}(x, y)$ is at hand from equation (55) and yields

$$
\begin{gathered}
f_{R}(x, y)= \\
-\frac{1}{2 \pi^{2}} \int_{0}^{\infty} \frac{d \tau}{\sqrt{1+\tau^{2}}} \text { P.V. } \int_{-\infty}^{\infty} d \xi\left(\frac{1}{\xi-x-y \tau}+\frac{1}{\xi-x+y \tau}\right) \frac{\partial}{\partial \xi} \mathcal{R}_{C C}^{\natural} g\left(\frac{R^{2}}{\xi}, \tau\right) .
\end{gathered}
$$

In Fig.10, the notations are the same as in Fig. 9. But here there are two "scanning" upper circular arcs, corresponding to two scattering angles $\omega$ and $(\pi-\omega)$. The moving detector at site $D$ register the sum of the scattered radiation flux densities at two different scattered energies corresponding to the previous scattering angles. 


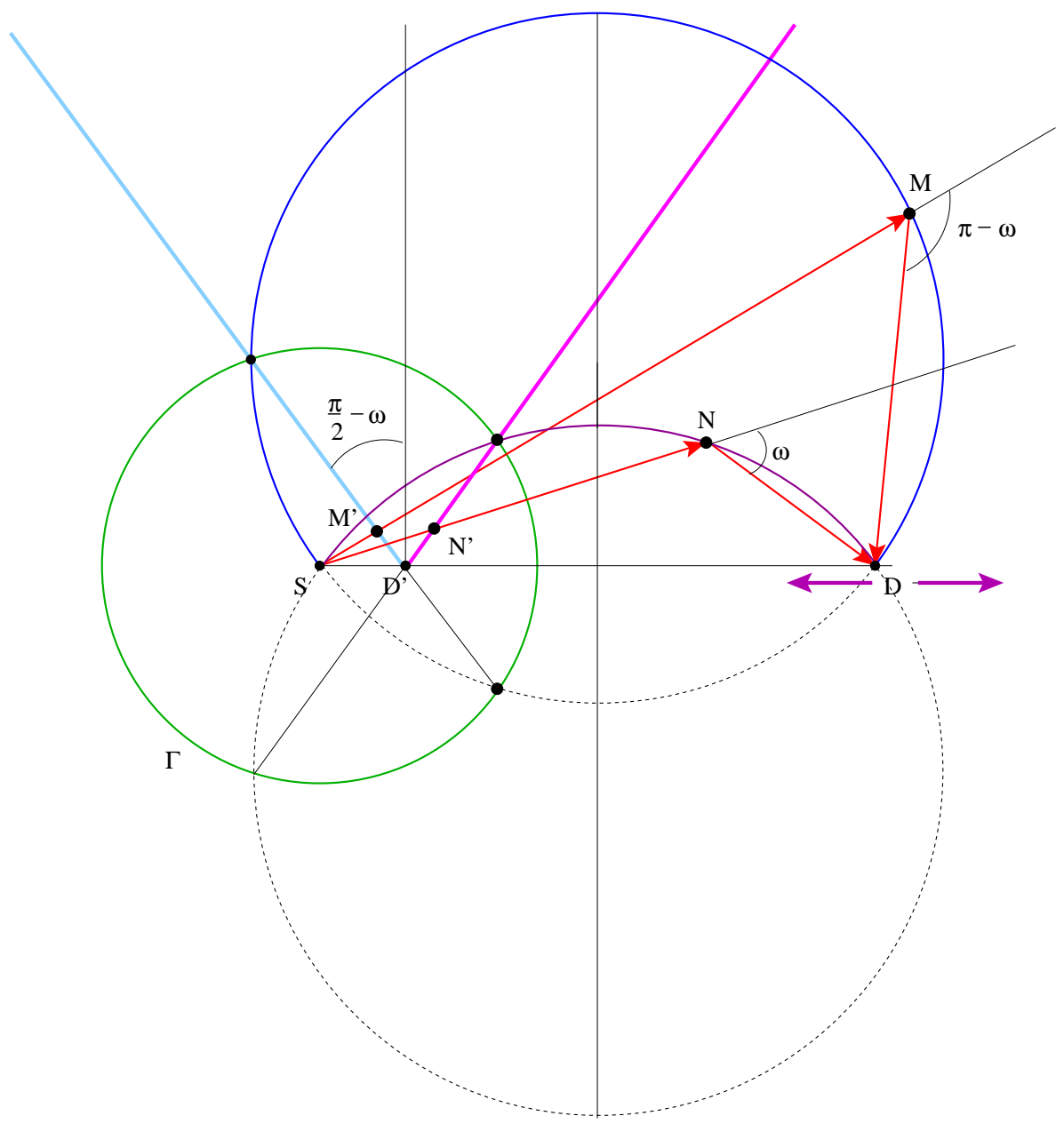

Figure 10. Double scanning in Norton CST - mapping to V-line scanning

Geometric inversion converts the pair of circular arcs ending at $S$ and at $D$ into the V-line with vertex at $D^{\prime}$, such that $S D \cdot S D^{\prime}=R^{2}$, as before. Image reconstruction goes through the inversion formula for the $\mathrm{V}$-line Radon transform and finally one recovers the actual electric charge density via equation (26). By construction, this new modality should show better robustness and sensitivity as the previous one. Comparative numerical tests on image quality are underway and should appear shortly.

\section{Conclusion and perspectives}

In this work we have derived some new properties of the V-line Radon transform introduced in 2006 [15] and studied in details in [17]. The first main result consists of its equivalence with the standard Radon transform, thus illustrating the fact that a large number of generalized Radon transforms are connected to the standard Radon transform, see e.g. $[30,38,40]$. Note that the Broken-line Radon transform is also shown to be mapped back to the standard Radon transform in [22]. The second result 
is its connection to a Radon transform on a pair of supplementary circular arcs, which interestingly leads naturally the way to a new modality of a way to do Compton scatter tomography, which was advocated long ago by S J Norton [34]. The interplay between these Radon transforms thanks to geometric inversion is one of the most intriguing and fruitful features in integral geometry. We hope to extend this approach to other classes of Radon transforms and find new applications in mathematics, physics as well as in imaging science.

\section{References}

[1] Radon J 1917 Über die Bestimmung von Funktionnen durch ihre Integralwerte längs gewisser Mannigfaltikeiten Ber.Verh.Sachs.Akad.Wiss. Leipzig-Math.-Natur.Kl. 69 262-77

[2] John F 1955 Planes Waves and Spherical Waves Applied to Partial Differential Equations (New York: Interscience).

[3] Militello B, Man'ko V I, Man'ko M A and Messina 2009 A Radon transform as a set of probability distributions Phys. Scr. $\mathbf{8 0} 058102$

[4] Gelfand I M 1960 Integral geometry and its relations to the theory of group representation Russian Math. Surveys 15 143-51

[5] Saygili K 2010 Trkalian fields and Radon transformation J. Math. Phys. 51, 033513

[6] Asorey M, Facchi P, Man'ko V I, Marmo G, Pascazio S and Sudarshan E G C 2007 Radon transform on the cylinder and tomography of a particle on the circle Physical Review A76 012117

[7] Wünche A 1997 Radon transform and pattern functions in quantum tomography, Journal of Modern Optics44 2293-331

[8] Mane V, Peggs S and Wei J 1997 Radon reconstruction in longitudinal space, Proceedings Particle Accelerator Conference, Vancouver BC Canada, Vol. 2 2158-60

[9] Barrett H H 1984 The Radon Transform and its Applications in Progress in Optics, Ed. E. Wolf, 21 219-86

[10] Bracewell R N 1956 Strip integration in radio astronomy Austr. J. Phys. 9 198-217

[11] Kershaw D 1970 The determination of the density distribution of a gas flowing in a pipe from mean density measurements J. Inst. Maths. Applics 6 111-4

[12] Chapman C H 1981 Generalized Radon transforms and slant stacks Geophysical Journal of the Royal Astronomical Society 66 445-53

[13] Basko R, Zeng G L and Gullberg G T 1997 Analytical reconstruction formula for the onedimensional Compton camera IEEE Tansactions on Nuclear Science, 44 1342-6

[14] Jung C Y and Moon S 2015 Inversion formulas for cone transforms arising in applications of Compton cameras Inverse Problems 30015006

[15] Nguyen M K and Truong T T 2006 Imagerie par rayonnement gamma diffus $\left(1^{\text {st }}\right.$ edition, Paris: Hermès Science Publications)

[16] Morvidone M, Nguyen M K, Truong T T and Zaidi H 2010 On the V-line Radon transform an its imaging applications, International Journal of Biomedical Imaging 2010208179

[17] Truong T T and Nguyen M K 2011 On new V-line Radon transforms in $\mathbb{R}^{2}$ and their inversion J.Phys.A:Math.Theor. 44075206

[18] Gouia-Zarrad R and Ambartsoumian G 2014 Exact inversion of the conical Radon transform with a fixed opening angle Inverse Problems 30, 045007

[19] Florescu L, Schotland J C and Markel V 2009 Single scattering optical tomography, Physical Review E 79036607

[20] Florescu L, Markel V and Schotland J C 2010 Single scattering optical tomography: simultaneous reconstruction of scattering and absorption Physical Review E $\mathbf{8 1} 016602$

[21] Florescu L, Markel V and Schotland J C 2011 Inversion formulas for the broken ray Radon transform Inverse Problems $\mathbf{2 7} 025002$

[22] Ambartsoumian G 2012 Inversion of the V-line Radon transform in a disc and its applications in imaging Computers and Mathematics with applications 64 260-5

[23] Ambartsoumian G and Moon S 2013 A series formula for inversion of the V-line Radon transform in a disc Computers and Mathematics with applications 66 1567-72

[24] Katsevich A and Krylov R 2013 Broken ray transform: inversion and a range condition Inverse Problems 29075008

[25] Krylov R and Katsevich A 2015 Inversion of the broken ray transform in the case of energydependent attenuation Phys. Med. Biol. 60, 4313 
[26] Zhao F, Schotland J C, and Markel V 2014 Inversion of the Star Transform Inverse Problems 30105001

[27] Toft P 1996 The Radon transform: theory and implementation Ph.D Thesis Technical University of Denmark, Lyngby

[28] Solmon D C 1976 The X-ray transform Journal of Mathematical Analysis and Applications56 61-83

[29] Tuy H K 1983 An inversion formula for cone-beam reconstruction SIAM Journal on Applied Mathematics43 542-52

[30] Cormack A M 1984 Radon's problem: old and new, SIAM AMS Proceedings, 14 33-9

[31] Cormack A M 1981 The Radon transform on a family of curves in the plane Proceedings of the American Mathematical Society, 83 325-30

[32] Nievergelt Y 1986 Elementary inversion of Radon's transform SIAM Review, 28 79-84

[33] Farmer F T and Collins M P 1971 A new approach to the determination of anatomical crosssections of the body by Compton scattering of gamma-rays Phys. Med. Biol., 16 577-86

[34] Norton S J 1994 Compton scattering tomography J. Appl. Phys., 76 2007-15

[35] Farmer F T and Collins M P 1974 A further appraisal of the Compton scattering method for determining anatomical cross-sections of the body Phys. Med. Biol.19 808-18

[36] Kondic N N, Jacobs A M and Ebert D 1983 Three-dimensional density field determination by external stationary detectors and gamma sources using selective scattering, in Thermalhydraulics of nuclear reactors, Vol.2, M. Merilo Ed., pp. 1443-1455, ANS, Santa Barbara California USA, January 11-14, 1983.

[37] Gautam S R, Hopkins F F, Klinksiek R and Morgan I L 1983 Compton interaction tomography IEEE Transactions on Nuclear Science, Vol. NS-30, 1680-4

[38] Nguyen M K and Truong T T 2010 Inversion of a new circular arc Radon transform for Compton scattering tomography Inverse Problems $\mathbf{2 6} 065005$

[39] Truong T T and Nguyen M K 2011 Radon transforms on generalized Cormack's curves and a new Compton scatter tomography modality, Inverse Problems $\mathbf{2 7} 125001$

[40] Truong T T 2014 On geometric aspects of circular arcs Radon transforms for Compton scatter tomography, Eurasian Journal of Mathematical and Computer Applications 2 40-69

[41] Truong T T and Nguyen M K 2012 Recent developments in Compton scatter tomography: Theory and Numerical simulations, pp. 101-28 in Numerical Simulation: From Theory to Industry, M Andriychuk Ed. (INTECH Rijeka, Croatia)

\section{Appendix: Derivation of the inverse formula in Cartesian coordinates}

For the convenience of the reader, we add a derivation of the full line Radon transform in Cartesian coordinates

$$
\mathcal{R} f(\xi, \tau)=\int_{\mathbb{R}} \sqrt{1+\tau^{2}} d y f(\xi-y \tau, y) .
$$

Introduce the Fourier representation

$$
f(x, y)=\int_{\mathbb{R}^{2}} d p d q e^{2 i \pi(p x+q y)} \widetilde{f}(p, q) .
$$

The result is the following

$$
\begin{aligned}
\frac{\mathcal{R} f(\xi, \tau)}{\sqrt{1+\tau^{2}}} & =\int_{\mathbb{R}} d y \int_{\mathbb{R}^{2}} d p d q e^{2 i \pi(p(\xi-y \tau)+q y)} \tilde{f}(p, q) \\
& =\int_{\mathbb{R}^{2}} d p d q \widetilde{f}(p, q) e^{2 i \pi p \xi} \int_{\mathbb{R}} d y e^{-2 i \pi y(p \tau-q)} \\
& =\int_{\mathbb{R}^{2}} d p d q \tilde{f}(p, q) e^{2 i \pi p \xi} \delta(p \tau-q) \\
& =\int_{\mathbb{R}} d p \widetilde{f}(p, p \tau) e^{2 i \pi p \xi}
\end{aligned}
$$

Now perform Fourier transform in $\xi$ on this last equation to get

$$
\int_{\mathbb{R}} d \xi e^{-2 i \pi \xi P} \frac{\mathcal{R} f(\xi, \tau)}{\sqrt{1+\tau^{2}}}=\tilde{f}(P, P \tau) .
$$


To reconstruct $f(x, y)$ use its Fourier representation, which requires $\widetilde{f}(p, q)$. But we only have $\widetilde{f}(p, p \tau)$. So we set $q=p \tau$, and move to variables $(\tau, q)$ instead of $(p, q)$, via the change of variables

$$
p=\frac{q}{\tau}, \quad \text { and } \quad q=q .
$$

The jacobian is

$$
d p d q=\left|\begin{array}{ll}
\frac{\partial p}{\partial \tau} & \frac{\partial p}{\partial q} \\
\frac{\partial q}{\partial \tau} & \frac{\partial q}{\partial q}
\end{array}\right| d \tau d q=\left|\begin{array}{cc}
-\frac{q}{\tau^{2}} & \frac{1}{\tau} \\
0 & 1
\end{array}\right| d \tau d q=\frac{|q|}{\tau^{2}} d \tau d q .
$$

Thus

$$
f(x, y)=\int_{\mathbb{R}^{2}} \frac{d \tau d \xi}{\tau^{2} \sqrt{1+\tau^{2}}} \mathcal{R} f(\xi, \tau) \int_{\mathbb{R}} d q|q| e^{2 i \pi\left(\frac{x}{\tau}+y-\frac{\xi}{\tau}\right)} .
$$

Performing the $q$-integral, we get

$$
\int_{\mathbb{R}} d q|q| e^{2 i \pi\left(\frac{x}{\tau}+y-\frac{\xi}{\tau}\right)}=-\frac{1}{2 \pi^{2}} \frac{1}{\left(\frac{x}{\tau}+y-\frac{\xi}{\tau}\right)^{2}},
$$

inserting in the expression of $f(x, y)$,

$$
f(x, y)=-\frac{1}{2 \pi^{2}} \text { P.V. } \int_{\mathbb{R}^{2}} \frac{d \tau d \xi}{\sqrt{1+\tau^{2}}} \frac{\mathcal{R} f(\xi, \tau)}{(x+\tau y-\xi)^{2}} .
$$

By partial integration on $d \xi$, we find

$$
f(x, y)=-\frac{1}{2 \pi^{2}} \text { P.V. } \int_{\mathbb{R}^{2}} \frac{d \tau d \xi}{\sqrt{1+\tau^{2}}} \frac{1}{(x+\tau y-\xi)} \frac{\partial}{\partial \xi} \mathcal{R} f(\xi, \tau),
$$

which is the formula of Cormack in [30], which differs from a sign with the result of [32]. 